\title{
Planet Candidates from K2 Campaigns 5-8 and Follow-up Optical Spectroscopy
}

\author{
Erik A. Petigura ${ }^{1,9}$ (1) Ian J. M. Crossfield ${ }^{2,3}$, Howard Isaacson ${ }^{4}$ (1), Charles A. Beichman ${ }^{5}$, Jessie L. Christiansen ${ }^{5}$ (1),

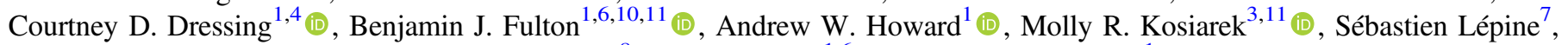 \\ Joshua E. Schlieder ${ }^{8}$, Evan Sinukoff ${ }^{1,6}{ }_{(\mathbb{D})}$, and Samuel W. Yee ${ }^{1}$ (D) \\ ${ }^{1}$ California Institute of Technology, 1200 E California Boulevard, Pasadena, CA, 91125, USA; petigura@ caltech.edu \\ ${ }^{2}$ Department of Physics, Massachusetts Institute of Technology, 77 Massachusetts Avenue, Cambridge, MA, USA \\ ${ }^{3}$ University of California Santa Cruz, Santa Cruz, CA, 95064, USA \\ ${ }^{4}$ Department of Astronomy, University of California, 501 Campbell Hall \#3411, Berkeley, CA 94720, USA \\ ${ }^{5}$ NASA Exoplanet Science Institute \& Infrared Processing and Analysis Center, California Institute of Technology, Pasadena, CA, 91125, USA \\ ${ }^{6}$ Institute for Astronomy, University of Hawai'i, Honolulu, HI 96822, USA \\ ${ }^{7}$ Department of Physics \& Astronomy, Georgia State University, 25 Park Place NE \#605, Atlanta, GA, 30303, USA \\ ${ }^{8}$ NASA Goddard Space Flight Center, 8800 Greenbelt Road, Greenbelt, MD 20771, USA \\ Received 2017 September 22; revised 2017 November 14; accepted 2017 November 14; published 2017 December 15
}

\begin{abstract}
We present 151 planet candidates orbiting 141 stars from $K 2$ campaigns 5-8 (C5-C8), identified through a systematic search of $K 2$ photometry. In addition, we identify 16 targets as likely eclipsing binaries, based on their light curve morphology. We obtained follow-up optical spectra of 105/141 candidate host stars and 8/16 eclipsing binaries to improve stellar properties and to identify spectroscopic binaries. Importantly, spectroscopy enables measurements of host star radii with $\approx 10 \%$ precision, compared to $\approx 40 \%$ precision when only broadband photometry is available. The improved stellar radii enable improved planet radii. Our curated catalog of planet candidates provides a starting point for future efforts to confirm and characterize $K 2$ discoveries.
\end{abstract}

Key words: planetary systems - planets and satellites: detection - planets and satellites: fundamental parameters stars: abundances - stars: fundamental parameters - techniques: spectroscopic

Supporting material: machine-readable tables

\section{Introduction}

NASA's Kepler Space Telescope, operating in its prime mission (2009-2013; Borucki et al. 2010), shed light on many fundamental properties of exoplanets. Among these are the occurrence of planets as small as Earth around Sun-like and low-mass stars (e.g., Petigura et al. 2013; Dressing \& Charbonneau 2015) and the diversity of planetary bulk compositions (Marcy et al. 2014; Weiss \& Marcy 2014; Rogers 2015) extending down to Earth-size (e.g., Howard et al. 2013; Jontof-Hutter et al. 2015).

Now operating in its two-wheel K2 mode (Howell et al. 2014), Kepler observes a different region of sky every three months. $K 2$ is conducting a wider, more shallow survey that complements the narrow, deep survey of the prime mission. Among its many accomplishments to date, $K 2$ has significantly increased the number of transiting planets around moderately bright stars (Crossfield et al. 2016), which will enable more detailed studies of exoplanet bulk composition using precision radial velocity facilities. $K 2$ has also revealed planets around newborn stars (David et al. 2016; Mann et al. 2016) and planets around white-dwarfs (Vanderburg et al. 2015). Due to community-driven target selection, a large fraction of the $K 2$ targets are M-dwarfs, resulting in the detection of planets in or near the habitable zone (e.g., Crossfield et al. 2015; Montet et al. 2015; Petigura et al. 2015; Schlieder et al. 2016).

In this paper, we provide a catalog of planet candidates and eclipsing binaries from the second year of $K 2$ operations,

\footnotetext{
${ }^{9}$ Hubble Fellow.

${ }^{10}$ Texaco Fellow.

${ }^{11}$ NSF Graduate Research Fellow.
}

corresponding to campaigns 5-8 (C5-C8). Section 2 presents our methodology for correcting spacecraft systematics in $K 2$ photometry and identifying planet candidates. In Section 3, we describe our spectroscopic follow-up program and present refined stellar parameters enabled by these spectra. We present our catalog of planet candidates and eclipsing binaries in Section 4 and summarize our findings in Section 5.

\section{Identifying Planet Candidates}

\subsection{Photometry}

During its prime mission, Kepler achieved photometric precisions of $\approx 40 \mathrm{ppm}$ on $6.5 \mathrm{hr}$ timescales (Christiansen et al. 2012) for targets of $\approx 12 \mathrm{mag}$ in the Kepler bandpass (i.e., $K p \approx 12 \mathrm{mag}$ ). For many stars, photometric precision was limited by intrinsic stellar variability rather than photon-limited or instrumental errors. This exquisite precision was due in large part to stable pointing enabled by four (and later three) reaction wheels, which stabilized the telescope against solar radiation pressure across the three axes of the telescope. Photometry for individual target stars was extracted using stationary software apertures composed of integer numbers of connected pixels.

During $K 2$ operations, where the spacecraft uses the two remaining operational reaction wheels, solar radiation pressure causes drifts of $\sim 1$ pixel to occur on $\sim 6 \mathrm{hr}$ timescales. As stars drift across the CCD, variations in pixel sensitivities and variable aperture losses result in apparent brightness variations.

Several techniques have been developed to correct for the position-dependent brightness variations due to the unstable platform of K2. A non-exhaustive list includes $\mathrm{k} 2 \mathrm{sff}$ (Vanderburg \& Johnson 2014), k2phot (Crossfield et al. 2015, 2016; Petigura et al. 2015), and k2sc (Aigrain 
et al. 2015), which model stellar brightness as a function of spacecraft orientation with a function and remove it from the light curve. The everest package (Luger et al. 2016) builds on the pixel-level decorrelation approach developed for Spitzer (Deming et al. 2015) and decorrelates against the pixel-by-pixel photometric time series.

We generated light curves for 87913 stars observed by $K 2$ during $\mathrm{C} 5-\mathrm{C} 8$ using the publicly available $\mathrm{k} 2$ phot Python package. $^{12}$ The general methodology is described in previous works (Crossfield et al. 2015, 2016; Petigura et al. 2015). However, due to the evolving nature of $K 2$ systematics, we have continued to adapt and refine $\mathrm{k} 2$ phot and summarize the methodology below.

As systematics in the photometry are largely due to pointing drifts, accurate knowledge of the spacecraft orientation is important for removing orientation-dependent systematics. We characterize the time-dependent orientation of the Kepler spacecraft by analyzing the positions of $\approx 100$ bright but unsaturated stars having $K p \approx 12 \mathrm{mag}$ on a representative output channel of the Kepler CCD. ${ }^{13}$ For each long-cadence integration, we solve for the affine transformation that maps that frame to an arbitrary reference frame. We then use the sequence of affine transformations to transform a reference pixel coordinate on a reference frame ${ }^{14}$ to the pixel coordinate on all other frames in the campaign.

We extract photometry using stationary apertures. Aperture size is described by a single variable $N_{\text {pix }}$, the number of pixels in the aperture. Apertures are constructed to accommodate image motion during a campaign. We construct apertures using a composite image constructed from the 90th percentile intensity value of all frames in a campaign. Because the stars move during $K 2$ observations, the 90th percentile image is smeared out and the apertures constructed from this image accommodate the drifts, mitigating severe aperture losses. The apertures are then constructed by selecting the pixel closest to the expected position of the target star, as predicted by the WCS coordinates provided by the $K 2$ project. Additional pixels are added iteratively by selecting the brightest pixel touching the current mask.

During the photometric extraction, we search for the aperture size $N_{\text {pix,min }}$ that minimizes noise on three-hour times scales. This aperture size strikes a balance between the desire to minimize systematic noise, which grows with decreasing aperture size, and background noise, which grows with increasing aperture size. We select an initial size $N_{\text {pix,0 }}$, which is motivated by previous analyses of stars with similar $K p$. We then try six logarithmically spaced $N_{\text {pix }}$ between $N_{\text {pix, } 0} / 4$ and $N_{\text {pix }, 0} \times 4$, which samples a curve describing noise as a function of $N_{\text {pix }}$. We find $N_{\text {pix,min }}$ using up to three iterations of Newton's method. While testing different aperture sizes, we constrain $N_{\text {pix }}$ to be between nine pixels and the total number of pixels in the target pixel file.

After extraction of the photometry, we have a sequence of flux as a function of $x, y$, and $t$. We model out changes in flux that correlate with changes in $x, y$, and $t$ using a Gaussian process with a squared-exponential covariance kernel, which is characterized by the following seven hyper-parameters $A_{x}, l_{x}$,

\footnotetext{
12 https://github.com/petigura/k2phot (commit a0d507).

13 The Kepler CCD contains 84 output channels (Kepler Instrument Handbook; Van Cleve et al. 2016), of which 76 were operational during C5-C8. An additional module (4 output channels) failed during $\mathrm{C} 10$.

14 For example, a pixel on row 500, column 500, and frame 2000.
}

$A_{y}, l_{y}, A_{t}, l_{t}$, and $\sigma$. Here, $A$ corresponds to the amplitude of the $\mathrm{GP}, l$ corresponds to characteristic length scale, and $\sigma$ accounts for a white noise component. Choosing the appropriate hyperparameters can be computationally intensive on a star-by-star basis. We therefore adopt a scheme from Aigrain et al. (2015), which optimizes the hyper-parameters on a subset of the photometry using a differential evolution global optimizer (Storn \& Price 1997).

We produced light curves for 87913 stars in C5-C8, which are available on the Exoplanet Follow-up Observing Program (ExoFOP) website. ${ }^{15}$ Along with the photometry, we included photometric diagnostic plots showing the extraction aperture and resulting detrended light curve. Figure 1 shows these diagnostic plots for an example planet candidate around EPIC211736671.

\subsection{Transiting Planet Search}

Our general transit search and vetting process is similar to that described in Crossfield et al. (2016). We give a brief summary of here. We searched the calibrated photometry for transiting planets using the publicly available TERRA algorithm. ${ }^{16}$ TERRA is a matched filter-based approach and is described by Petigura et al. (2013). TERRA convolves the photometry with a box-shaped approximation of a transit profile to compute a Single Event Statistic (SES) at every $K 2$ long-cadence measurement. The SES time series is phasefolded according to a finely spaced grid of trial periods $P$ and times of first transit $T_{0}$.

A classical matched-filter algorithm would then compute a Multiple Event Statistic (MES) by summing SES at each trial $\left(P, T_{0}\right)$, which is optimal given uncorrelated Gaussian noise. However, in $K 2$ photometry we observe more frequent nonGaussian anomalies relative to Kepler prime photometry due to the aggressive detrending that must be performed in order to remove the instrumental systematics described above. A traditional MES computation resulted in many spurious peaks with apparently high MES, but were later easily identifiable as anomalies through inspection. We address outliers by calculating MES only after removing the two highest SES peaks at each trial $\left(P, T_{0}\right)$. Spurious peaks due to the chance alignment of two outliers are eliminated. This nonlinear filter removes many spurious detections and eases the burden during manual vetting, described below. One consequence is that TERRA does not identify planets with one or two transits occurring in a $K 2$ campaign. Such transits are sometimes identified by eye, but we caution that many are likely overlooked. These events are especially amenable to searches by citizen scientists (see, e.g., Christiansen et al. 2017). ${ }^{17}$

TERRA identifies 1000 Threshold-Crossing Events (TCEs) per campaign. A TCE is a particular combination of $\left(P, T_{0}\right)$ that has MES that exceeds some threshold, but may not be an astrophysical transit. If a candidate has a periodic dimming of a consistent shape it is elevated to the status of a " $K 2$ Object of Interest" (K2OIs), which are consistent with an astrophysical transit or eclipse.

Our team visually inspects each K2OI to look for a robust indication that the target is an eclipsing binary. We look for secondary eclipses, which indicate that the transiting object is

\footnotetext{
15 https://exofop.ipac.caltech.edu/

16 https://github.com/petigura/terra (commit 9739e9).

17 https://www.zooniverse.org/projects/ianc2/exoplanet-explorers
} 

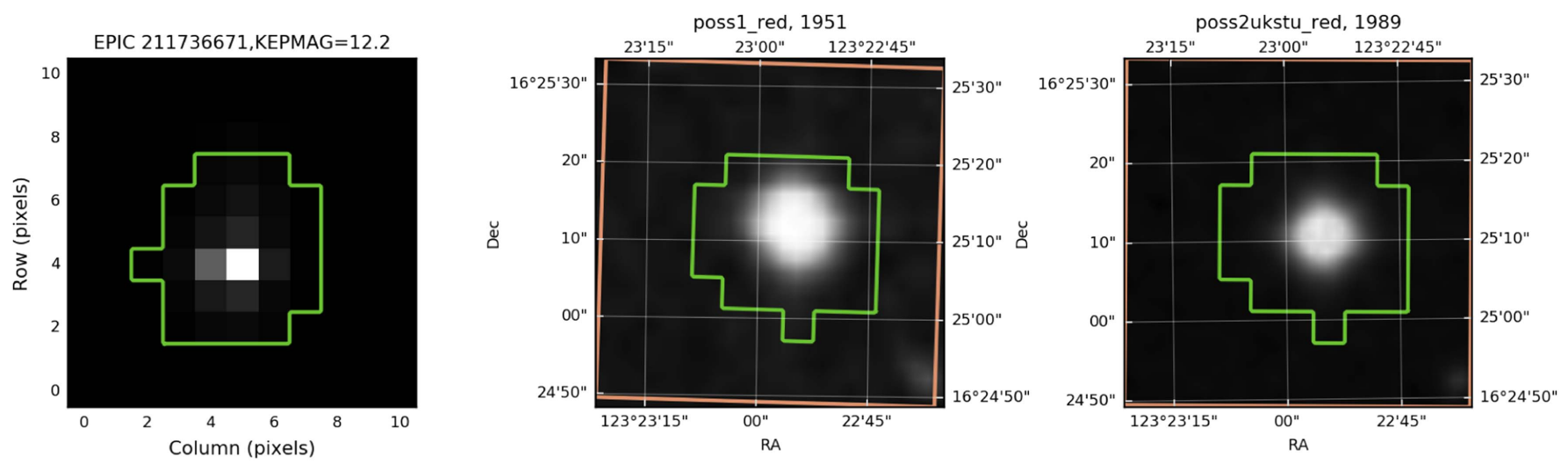

(a)
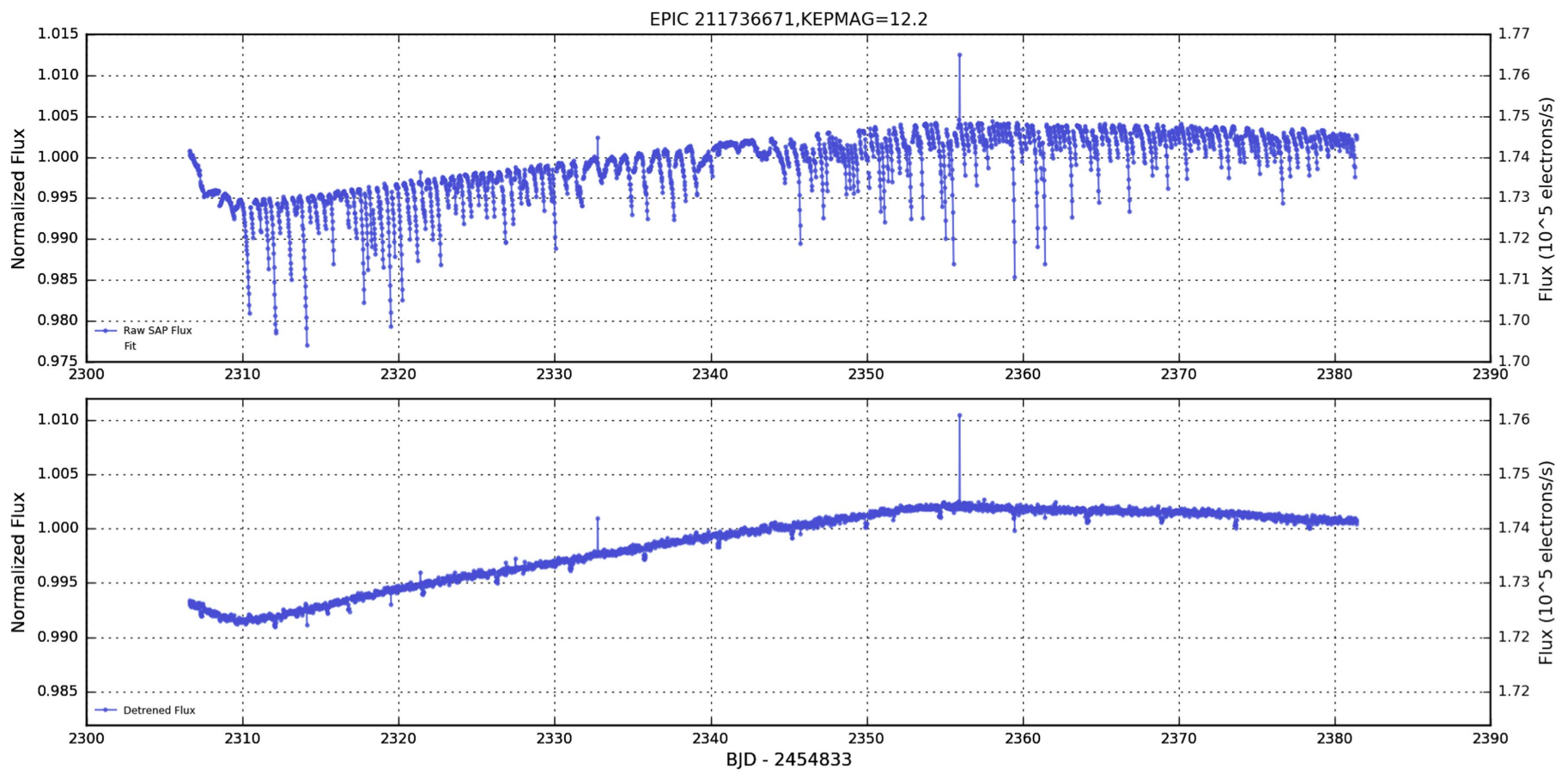

(b)

Figure 1. An example of the photometric diagnostic plots included as standard data products on the ExoFOP. Panel (a): three images of an example planet candidate (EPIC-211736671), observed in C5. Left: the median of all long-cadence C5 frames with the optimal extraction aperture shown in green. Center: the same region of sky as observed by the first Palomar Observatory Sky Survey (POSS-I). The orange region corresponds to the boundaries of the K2 frame. Right: the same region of sky as observed by POSS-II. Panel (b): aperture photometry before and after subtraction of our systematic noise model.

self-luminous and not a planet. Secondary eclipses associated with binaries with circular orbits are shifted in phase from the primary eclipse by $180^{\circ}$. We search for secondary eclipses at all phases to allow for eccentric orbits. We also look for obvious odd/even variations, which indicate that TERRA has identified a nearly circular EB at half the orbital period. We also identify stars that show variability that is phase-locked to the eclipse, which is a strong indicator of star-star modulation (ellipsoidal, reflection, or relativistic beaming).

Our eclipsing binary designation does not incorporate transit depth or whether the light curve is V-shaped. While these attributes are strong indicators of EB status, they are not conclusive. Planets transiting small M-dwarf stars can easily produce transits deeper than $1 \%$ and short period transits may appear V-shaped due to the 30-minute sampling of $K 2$. We defer a detailed false-positive calculation for a later paper.
In total, we identified $167 \mathrm{~K} 2 \mathrm{OIs}$, associated with 157 stars. Of these, 16/167 are likely eclipsing binaries, and we refer to the remaining 151 as planet candidates.

\subsection{Light Curve Fitting}

We fit the calibrated photometry according to the methodology of Crossfield et al. (2016). In brief, we used the publicly available batman light curve code (Kreidberg 2015) to generate model light curves that we then compared against the photometry. We first derived a maximum likelihood solution and then derived parameter uncertainties using Markov Chain Monte Carlo (MCMC). ${ }^{18}$

\footnotetext{
${ }^{18}$ Using the affine-invariant sampler of Goodman \& Weare (2010) as implemented in Python by Foreman-Mackey et al. (2015).
} 
Table 1

Planet Candidates

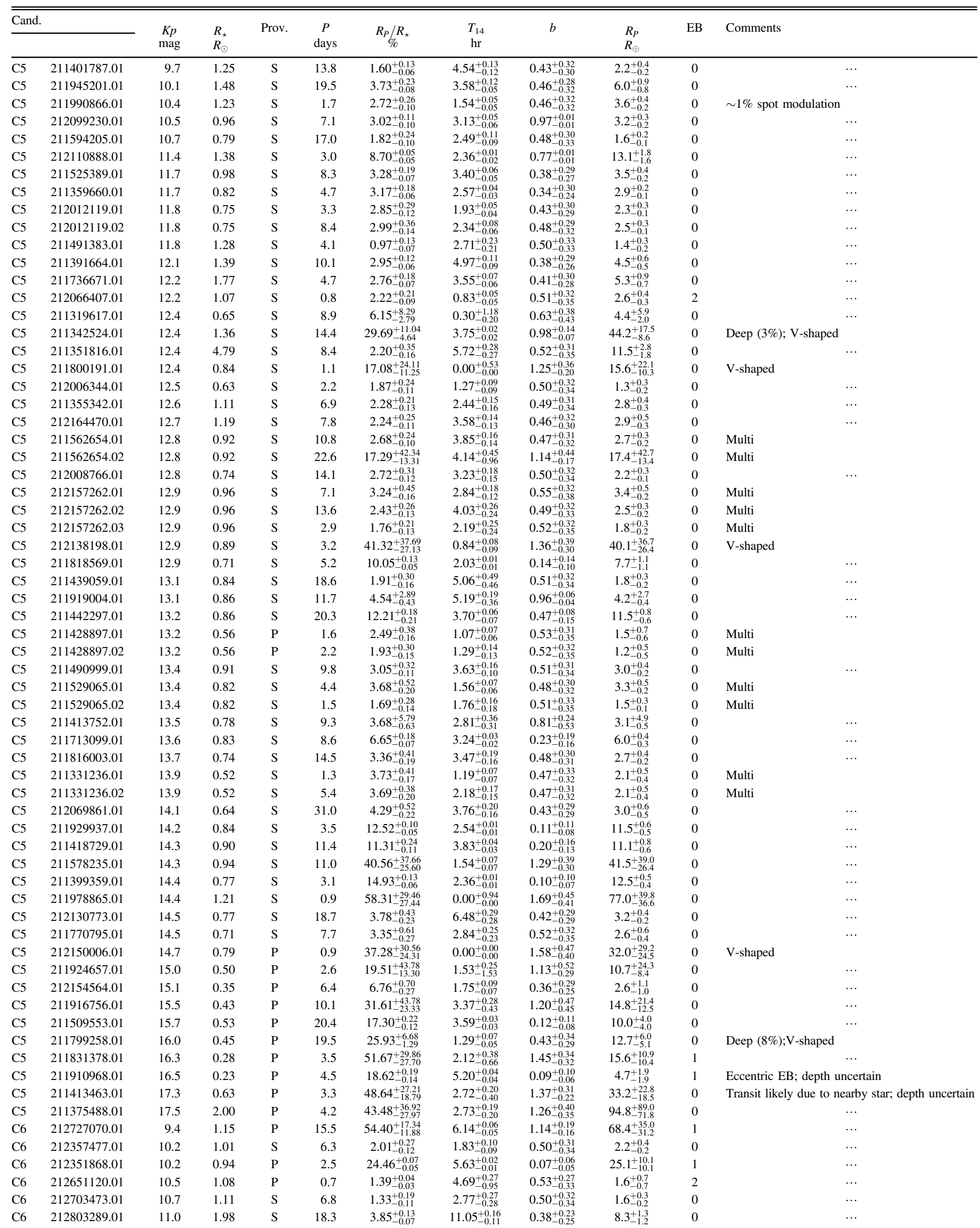


Table 1

(Continued)

\begin{tabular}{|c|c|c|c|c|c|c|c|c|c|c|c|}
\hline \multicolumn{2}{|c|}{ Cand. } & $\begin{array}{c}K p \\
\text { mag }\end{array}$ & $\begin{array}{l}R_{\star} \\
R_{\odot}\end{array}$ & Prov. & $\begin{array}{c}P \\
\text { days }\end{array}$ & & $\begin{array}{l}T_{14} \\
\mathrm{hr}\end{array}$ & $b$ & $\begin{array}{l}R_{P} \\
R_{\oplus} \\
\end{array}$ & EB & Comments \\
\hline C6 & 212773309.01 & 11.4 & 0.86 & $\mathrm{~S}$ & 4.7 & $24.44_{-0.28}^{+0.29}$ & $2.28_{-0.02}^{+0.02}$ & $0.69_{-0.02}^{+0.02}$ & $22.9_{-1.0}^{+1.3}$ & 1 & $\ldots$ \\
\hline C6 & 212577658.01 & 11.5 & 0.84 & $\mathrm{P}$ & 14.1 & $2.01_{-0.12}^{+0.29}$ & $2.99_{-0.12}^{+0.12}$ & $0.49_{-0.33}^{+0.023}$ & $1.8_{-0.7}^{+0.8}$ & 0 & $\ldots$ \\
\hline $\mathrm{C} 6$ & 212782836.01 & 11.6 & 0.76 & S & 7.1 & $1.26_{-0.09}^{+0.17}$ & $3.14_{-0.25}^{+0.26}$ & $0.49_{-0.33}^{+0.32}$ & $1.0_{-0.1}^{+0.1}$ & 0 & low $\mathrm{S} / \mathrm{N}$ \\
\hline C6 & 212521166.01 & 11.6 & 0.68 & S & 13.9 & $3.33_{-0.08}^{+0.22}$ & $3.20_{-0.05}^{+0.06}$ & $0.41_{-0.27}^{+0.28}$ & $2.5_{-0.1}^{+0.2}$ & 0 & $\cdots$ \\
\hline C6 & 212586030.01 & 11.7 & 3.83 & S & 7.8 & $37.28_{-26.58}^{+39.24}$ & $1.26_{-1.26}^{+0.52}$ & $1.36_{-0.29}^{+0.41}$ & $155.7_{-113.0}^{+165.8}$ & 0 & $\cdots$ \\
\hline C6 & 212300977.01 & 11.7 & 1.11 & $\mathrm{~S}$ & 4.5 & $12.23_{-0.02}^{+0.04}$ & $3.52_{-0.01}^{+0.01}$ & $0.07_{-0.05}^{+0.07}$ & $14.8_{-1.2}^{+1.7}$ & 0 & $\ldots$ \\
\hline C6 & 212779596.01 & 11.9 & 0.70 & $\mathrm{~S}$ & 7.4 & $3.94_{-0.16}^{+0.45}$ & $2.45_{-0.08}^{+0.11}$ & $0.48_{-0.33}^{+0.31}$ & $3.0_{-0.4}^{+0.6}$ & 0 & Multi \\
\hline C6 & 212779596.02 & 11.9 & 0.70 & $\mathrm{~S}$ & 3.2 & $2.45_{-0.13}^{+0.32}$ & $2.04_{-0.10}^{+0.10}$ & $0.52_{-0.35}^{+0.31}$ & $1.9_{-0.3}^{+0.4}$ & 0 & Multi \\
\hline C6 & 212735333.01 & 12.0 & 0.96 & $\mathrm{~S}$ & 8.4 & $2.41_{-0.15}^{+0.32}$ & $3.47_{-0.18}^{+0.19}$ & $0.49_{-0.33}^{+0.31}$ & $2.5_{-0.2}^{+0.4}$ & 0 & $\ldots$ \\
\hline C6 & 212679181.01 & 12.0 & 0.36 & $\mathrm{P}$ & 1.1 & $2.49_{-0.17}^{+0.33}$ & $0.49_{-0.05}^{+0.06}$ & $0.50_{-0.34}^{+0.33}$ & $1.0_{-0.4}^{+0.4}$ & 0 & $\ldots$ \\
\hline C6 & 212587672.01 & 12.2 & 0.95 & $\mathrm{~S}$ & 23.2 & $2.20_{-0.13}^{+0.26}$ & $3.23_{-0.16}^{+0.19}$ & $0.49_{-0.34}^{+0.32}$ & $2.3_{-0.2}^{+0.3}$ & 0 & $\ldots$ \\
\hline C6 & 212697709.01 & 12.2 & 1.20 & $\mathrm{~S}$ & 4.0 & $9.17_{-0.11}^{+0.11}$ & $1.83_{-0.03}^{+0.02}$ & $0.85_{-0.01}^{+0.01}$ & $12.0_{-1.3}^{+1.7}$ & 0 & WASP-157b \\
\hline C6 & 212394689.01 & 12.2 & 0.88 & $\mathrm{~S}$ & 6.7 & $2.71_{-0.09}^{+0.22}$ & $2.71_{-0.08}^{+0.09}$ & $0.46_{-0.31}^{+0.30}$ & $2.6_{-0.2}^{+0.3}$ & 0 & $\cdots$ \\
\hline C6 & 212689874.01 & 12.3 & 0.95 & S & 15.9 & $2.87_{-0.08}^{-0.16}$ & $4.84_{-0.12}^{+0.14}$ & $0.39_{-0.27}^{+0.31}$ & $3.0_{-0.2}^{+0.4}$ & 0 & $\ldots$ \\
\hline $\mathrm{C} 6$ & 212435047.01 & 12.4 & 1.10 & S & 1.1 & $1.32_{-0.10}^{+0.19}$ & $1.58_{-0.23}^{-0.21}$ & $0.52_{-0.35}^{+0.34}$ & $1.6_{-0.2}^{+0.3}$ & 0 & V-shaped; low S/N \\
\hline C6 & 212460519.01 & 12.4 & 0.66 & $\mathrm{~S}$ & 7.4 & $2.78_{-0.13}^{+0.31}$ & $2.63_{-0.08}^{+0.09}$ & $0.48_{-0.32}^{+0.28}$ & $2.0_{-0.3}^{+0.4}$ & 0 & $\ldots$ \\
\hline C6 & 212639319.01 & 12.5 & 0.81 & $P$ & 13.8 & $24.21_{-19.27}^{+44.69}$ & $1.77_{-0.18}^{+0.19}$ & $1.20_{-0.22}^{+0.46}$ & $21.4_{-19.1}^{+40.4}$ & 0 & $\ldots$ \\
\hline C6 & 212555594.02 & 12.5 & 0.83 & $\mathrm{P}$ & 4.2 & $1.68_{-0.17}^{+0.26}$ & $1.55_{-0.20}^{+0.20}$ & $0.50_{-0.34}^{+0.32}$ & $1.5_{-0.6}^{+0.7}$ & 0 & $\begin{array}{l}212555594.01 \text { is due to noise, } 212555594.02 \\
\text { looks transit-like }\end{array}$ \\
\hline C6 & 212428509.01 & 12.5 & 0.94 & S & 2.7 & $49.70_{-8.53}^{+8.63}$ & $2.09_{-0.19}^{+0.16}$ & $1.42_{-0.10}^{+0.10}$ & $50.7_{-9.7}^{+11.9}$ & 0 & Deep (1\%); V-shaped \\
\hline $\mathrm{C} 6$ & 212585579.01 & 12.6 & 1.09 & S & 3.0 & $38.76_{-24.16}^{+35.69}$ & $0.00_{-0.00}^{+0.00}$ & $1.45_{-0.30}^{+0.44}$ & $45.9_{-28.9}^{+42.6}$ & 0 & V-shaped \\
\hline C6 & 212691727.01 & 12.7 & 0.87 & $P$ & 12.9 & $23.20_{-0.49}^{+0.65}$ & $4.97_{-0.05}^{+0.06}$ & $0.19_{-0.13}^{+0.14}$ & $22.1_{-8.8}^{+8.8}$ & 1 & $\ldots$ \\
\hline C6 & 212572439.01 & 12.8 & 0.78 & $\mathrm{P}$ & 2.6 & $6.68_{-0.32}^{+0.40}$ & $1.69_{-0.04}^{+0.07}$ & $0.60_{-0.29}^{+0.16}$ & $5.7_{-2.3}^{+2.3}$ & 0 & $\begin{array}{l}\text { EPIC-212572452 }(K p=14.8) \text { in photometric } \\
\text { aperture }\end{array}$ \\
\hline C6 & 212756297.01 & 13.0 & 0.66 & $P$ & 1.3 & $15.99_{-0.01}^{+0.02}$ & $1.85_{-0.00}^{+0.00}$ & $0.03_{-0.02}^{+0.04}$ & $11.5_{-4.6}^{+4.6}$ & 0 & $\ldots$ \\
\hline C6 & 212580872.01 & 13.0 & 0.83 & $\mathrm{P}$ & 14.8 & $3.59_{-0.08}^{+0.16}$ & $4.32_{-0.07}^{+0.09}$ & $0.34_{-0.23}^{+0.27}$ & $3.3_{-1.3}^{+1.3}$ & 0 & $\ldots$ \\
\hline C6 & 212797028.01 & 13.1 & 0.94 & $\mathrm{P}$ & 30.0 & $14.81_{-0.24}^{+0.36}$ & $6.37_{-0.06}^{+0.06}$ & $0.85_{-0.01}^{+0.01}$ & $15.3_{-6.1}^{+6.1}$ & 0 & Deep ( 2\%); V-shaped \\
\hline C6 & 212443457.01 & 13.1 & 0.68 & $\mathrm{P}$ & 24.5 & $18.42_{-6.81}^{+29.25}$ & $8.49_{-0.21}^{+0.18}$ & $1.02_{-0.11}^{+0.34}$ & $13.6_{-7.4}^{+22.3}$ & 0 & $\begin{array}{l}\text { Deep (1\%); irregular transit shape; possible } \\
\text { hierarchical triple }\end{array}$ \\
\hline C6 & 212432685.01 & 13.1 & 1.06 & $\mathrm{P}$ & 0.5 & $1.69_{-0.09}^{+0.18}$ & $1.59_{-0.22}^{+0.12}$ & $0.48_{-0.33}^{+0.37}$ & $2.0_{-0.8}^{+0.8}$ & 0 & $\ldots$ \\
\hline C6 & 212294561.01 & 13.1 & 0.85 & $P$ & 2.8 & $21.48_{-16.50}^{+36.05}$ & $0.00_{-0.00}^{+0.00}$ & $1.39_{-0.29}^{+0.54}$ & $19.9_{-17.2}^{+34.3}$ & 1 & $\ldots$ \\
\hline C6 & 212418133.01 & 13.2 & 0.90 & $\mathrm{P}$ & 3.3 & $1.59_{-0.13}^{+0.23}$ & $3.48_{-0.34}^{+0.31}$ & $0.48_{-0.33}^{+0.33}$ & $1.6_{-0.6}^{+0.7}$ & 0 & $\ldots$ \\
\hline C6 & 212628098.01 & 13.3 & 0.67 & $P$ & 4.4 & $22.72_{-0.49}^{+0.52}$ & $1.63_{-0.03}^{+0.03}$ & $0.64_{-0.05}^{+0.04}$ & $16.6_{-6.7}^{+6.7}$ & 0 & Deep ( 5\%); V-shaped; spot mod. \\
\hline C6 & 212839127.01 & 13.3 & 1.05 & $\mathrm{P}$ & 20.6 & $43.10_{-11.79}^{+21.49}$ & $4.03_{-0.02}^{+0.02}$ & $1.11_{-0.15}^{+0.24}$ & $49.5_{-24.0}^{+31.6}$ & 0 & Deep ( 5\%); V-shaped; \\
\hline C6 & 212579164.01 & 13.6 & 0.83 & $P$ & 18.2 & $60.70_{-8.51}^{+14.10}$ & $3.59_{-0.07}^{+0.08}$ & $0.74_{-0.15}^{+0.18}$ & $55.0_{-23.3}^{+25.4}$ & 0 & V-shaped; Deep ( 20\%) \\
\hline C6 & 212751916.01 & 13.9 & 0.90 & $P$ & 15.7 & $24.26_{-19.77}^{+46.48}$ & $2.71_{-0.47}^{+0.54}$ & $1.19_{-0.41}^{+0.48}$ & $23.7_{-21.5}^{+46.4}$ & 1 & $\ldots$ \\
\hline C6 & 212570977.01 & 13.9 & 0.87 & $\mathrm{P}$ & 8.9 & $15.14_{-0.10}^{+0.10}$ & $4.22_{-0.03}^{+0.03}$ & $0.43_{-0.05}^{+0.04}$ & $14.4_{-5.8}^{+5.8}$ & 0 & $\cdots$ \\
\hline C6 & 212757039.01 & 14.4 & 0.80 & $P$ & 4.5 & $16.06_{-0.09}^{+0.09}$ & $3.34_{-0.02}^{+0.02}$ & $0.79_{-0.01}^{+0.01}$ & $14.0_{-5.6}^{+5.6}$ & 1 & $\ldots$ \\
\hline C6 & 212311834.01 & 14.7 & 0.76 & $\mathrm{P}$ & 17.8 & $57.91_{-15.49}^{+21.67}$ & $2.81_{-0.02}^{+0.02}$ & $1.06_{-0.21}^{+0.25}$ & $47.8_{-230}^{+26.2}$ & 0 & V-shaped; Deep $(\sim 10 \%)$ \\
\hline C6 & 212554013.01 & 14.7 & 0.75 & $\mathrm{P}$ & 3.6 & $11.17_{-0.21}^{+0.38}$ & $2.19_{-0.03}^{+0.06}$ & $0.37_{-0.24}^{+0.19}$ & $9.1_{-3.7}^{+3.7}$ & 0 & $\ldots$ \\
\hline C6 & 212679798.01 & 14.8 & 0.67 & $\mathrm{P}$ & 1.8 & $36.83_{-7.89}^{+3.21 .79}$ & $2.19_{-0.04}^{+0.04}$ & $0.86_{-0.17}^{+0.44}$ & $27.1_{-12.3}^{+25.8}$ & 1 & $\ldots$ \\
\hline $\mathrm{C} 6$ & 212773272.01 & 15.0 & 0.25 & $P$ & 4.7 & $19.87_{-0.68}^{+1.00}$ & $2.05_{-0.11}^{+0.14}$ & $0.32_{-0.22}^{+0.25}$ & $5.4_{-2.2}^{+2.2}$ & 0 & Deep $(\sim 5 \%)$; \\
\hline C6 & 212421319.01 & 16.4 & 0.80 & $P$ & 5.5 & $54.69_{-28.57}^{+31.61}$ & $7.01_{-2.08}^{+0.99}$ & $1.41_{-0.38}^{+0.38}$ & $48.0_{-31.6}^{+33.7}$ & 1 & 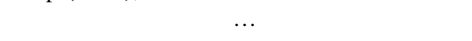 \\
\hline $\mathrm{C} 6$ & 212757601.01 & 16.8 & 0.75 & $P$ & 1.0 & $43.93_{-24.84}^{+34.93}$ & $0.96_{-0.96}^{+0.63}$ & $1.40_{-0.36}^{+0.068}$ & $36.1_{-25.0}^{+3.1}$ & 0 & $\ldots$ \\
\hline $\mathrm{C} 7$ & 218541396.01 & 10.0 & 1.18 & $P$ & 1.0 & $52.48_{-25.10}^{+29.45}$ & $0.00_{-0.00}^{+1.21}$ & $1.61_{-0.39}^{+0.46}$ & $67.3_{-42.0}^{+46.4}$ & 1 & Deep ( 2\%); V-shaped; \\
\hline $\mathrm{C} 7$ & 213920015.01 & 10.0 & 0.87 & S & 1.5 & $1.04_{-0.05}^{+0.09}$ & $1.46_{-0.12}^{+0.10}$ & $0.50_{-0.34}^{+0.33}$ & $1.0_{-0.1}^{+0.1}$ & 0 & $\ldots$ \\
\hline $\mathrm{C} 7$ & 218711655.01 & 11.3 & 1.46 & S & 1.2 & $7.77_{-3.24}^{+4.84}$ & $0.00_{-0.00}^{+0.00}$ & $1.29_{-0.11}^{+0.10}$ & $12.3_{-5.3}^{+7.9}$ & 0 & 2 stars in aper; possible blended EB \\
\hline $\mathrm{C} 7$ & 218916923.01 & 11.5 & 0.93 & $\mathrm{~s}$ & 28.4 & $9.36_{-0.03}^{+0.03}$ & $4.94_{-0.02}^{+0.02}$ & $0.08_{-0.06}^{+0.08}$ & $9.5_{-0.5}^{+0.6}$ & 0 & $\ldots$ \\
\hline C7 & 214611894.01 & 11.9 & 1.21 & $\mathrm{~S}$ & 21.6 & $15.57_{-0.06}^{+0.13}$ & $4.07_{-0.02}^{+0.02}$ & $0.11_{-0.08}^{+0.10}$ & $20.5_{-2.1}^{+2.8}$ & 0 & Deep ( 3\%); flat-bottom \\
\hline C7 & 213546283.01 & 12.0 & 1.10 & $\mathrm{~s}$ & 9.8 & $2.77_{-0.12}^{+0.24}$ & $2.98_{-0.14}^{+0.15}$ & $0.48_{-0.33}^{+0.31}$ & $3.3_{-0.4}^{+0.6}$ & 0 & $\ldots$ \\
\hline C7 & 215938010.01 & 12.1 & 1.64 & $\mathrm{~S}$ & 1.2 & $6.91_{-3.22}^{+5.16}$ & $0.00_{-0.00}^{+0.00}$ & $1.28_{-0.12}^{+0.12}$ & $12.4_{-5.9}^{+9.4}$ & 0 & $\ldots$ \\
\hline $\mathrm{C} 7$ & 216494238.01 & 12.3 & 1.45 & $\mathrm{~S}$ & 19.9 & $5.37_{-0.08}^{+0.14}$ & $8.19_{-0.07}^{+0.12}$ & $0.37_{-0.25}^{+0.19}$ & $8.5_{-1.0}^{+1.2}$ & 0 & $\ldots$ \\
\hline $\mathrm{C} 7$ & 219388192.01 & 12.3 & 1.00 & S & 5.3 & $8.79_{-0.05}^{+0.09}$ & $3.30_{-0.02}^{+0.03}$ & $0.16_{-0.11}^{+0.15}$ & $9.6_{-0.5}^{+0.0}$ & 0 & $\ldots$ \\
\hline $\mathrm{C} 7$ & 218621322.01 & 12.4 & 1.00 & S & 11.6 & $20.20_{-15.46}^{+46.42}$ & $3.90_{-3.90}^{+0.73}$ & $1.17_{-0.21}^{+0.51}$ & $21.9_{-16.9}^{+50.5}$ & 0 & Variable depth, poss. contam. nearby source $\%$ \\
\hline $\mathrm{C} 7$ & 216892056.01 & 12.5 & 0.42 & $\mathrm{P}$ & 2.8 & $4.13_{-0.21}^{+0.43}$ & $0.50_{-0.03}^{+0.06}$ & $0.46_{-0.31}^{+0.32}$ & $1.9_{-0.8}^{+0.8}$ & 0 & $\ldots$ \\
\hline $\mathrm{C} 7$ & 217192839.01 & 12.6 & 0.70 & S & 16.0 & $2.60_{-0.15}^{+0.31}$ & $3.07_{-0.20}^{+0.22}$ & $0.48_{-0.32}^{+0.32}$ & $2.0_{-0.3}^{+0.4}$ & 0 & $\ldots$ \\
\hline $\mathrm{C} 7$ & 218131080.01 & 12.7 & 1.22 & S & 3.1 & $5.87_{-0.04}^{+0.08}$ & $4.48_{-0.02}^{+0.03}$ & $0.21_{-0.15}^{+0.18}$ & $7.8_{-0.6}^{+0.9}$ & 0 & HAT-S-12 b \\
\hline $\mathrm{C} 7$ & 216468514.01 & 12.7 & 1.73 & S & 3.3 & $7.64_{-0.18}^{+0.19}$ & $2.98_{-0.05}^{+0.06}$ & $0.50_{-0.21}^{+0.13}$ & $14.4_{-1.9}^{+2.2}$ & 0 & $\ldots$ \\
\hline $\mathrm{C} 7$ & 219420915.01 & 12.8 & 1.30 & S & 0.5 & $18.38_{-4.18}^{+7.76}$ & $0.00_{-0.00}^{+0.00}$ & $1.31_{-0.10}^{+0.11}$ & $26.1_{-6.6}^{+11.6}$ & 0 & V-shaped \\
\hline $\mathrm{C} 7$ & 216334329.01 & 12.9 & 1.54 & S & 28.1 & $39.99_{-26.41}^{+40.06}$ & $3.62_{-0.18}^{+0.20}$ & $1.35_{-0.28}^{+0.41}$ & $67.3_{-45.1}^{+68.1}$ & 0 & $\ldots$ \\
\hline $\mathrm{C} 7$ & 219256848.01 & 13.1 & 11.57 & S & 20.9 & $46.86_{-28.86}^{+35.73}$ & $3.49_{-0.18}^{+0.19}$ & $1.39_{-0.31}^{+0.37}$ & $591.1_{-367.2}^{+453.8}$ & 0 & $\ldots$ \\
\hline $\mathrm{C} 7$ & 217671466.01 & 13.1 & 1.94 & $\mathrm{~s}$ & 1.9 & $8.13_{-0.04}^{+0.09}$ & $3.56_{-0.01}^{+0.02}$ & $0.13_{-0.09}^{+0.12}$ & $17.2_{-2.2}^{+2.9}$ & 0 & Known HAT-S system \\
\hline $\mathrm{C} 7$ & 215389654.01 & 13.2 & 0.97 & S & 23.5 & $17.41_{-0.11}^{+0.27}$ & $8.58_{-0.12}^{+0.11}$ & $0.20_{-0.14}^{+0.16}$ & $18.4_{-1.2}^{+1.8}$ & 0 & Deep ( $\sim \%$ ); flat-bottom \\
\hline C7 & 213840781.01 & 13.7 & 0.76 & $P$ & 12.4 & $43.63_{-15.25}^{+26.02}$ & $2.86_{-0.12}^{+0.09}$ & $1.03_{-0.23}^{+0.31}$ & $36.3_{-19.3}^{+26.0}$ & 0 & Deep ( 4\%); V-shaped; \\
\hline $\mathrm{C} 7$ & 216414930.01 & 13.7 & 0.89 & $P$ & 3.6 & $10.52_{-0.03}^{+0.05}$ & $4.42_{-0.01}^{+0.01}$ & $0.07_{-0.05}^{+0.08}$ & $10.2_{-4.1}^{+4.1}$ & 0 & $\ldots$ \\
\hline C7 & 215358983.01 & 13.8 & 1.10 & S & 6.4 & $12.79_{-0.07}^{+0.13}$ & $6.12_{-0.04}^{+0.04}$ & $0.11_{-0.08}^{+0.11}$ & $15.3_{-1.4}^{+1.8}$ & 0 & Deep ( $\sim 2 \%)$; flat-bottomed \\
\hline
\end{tabular}


Table 1

(Continued)

\begin{tabular}{|c|c|c|c|c|c|c|c|c|c|c|c|}
\hline \multicolumn{2}{|c|}{ Cand. } & $\begin{array}{c}K p \\
\text { mag }\end{array}$ & $\begin{array}{l}R_{\star} \\
R_{\odot} \\
\end{array}$ & Prov. & $\begin{array}{c}P \\
\text { days }\end{array}$ & $R_{P} \int_{\%} R_{\star}$ & $\begin{array}{c}T_{14} \\
\mathrm{hr}\end{array}$ & $b$ & $\begin{array}{l}R_{P} \\
R_{\oplus} \\
\end{array}$ & EB & Comments \\
\hline C7 & 213703832.01 & 13.9 & 0.75 & $\mathrm{P}$ & 0.5 & $4.09_{-0.51}^{+0.96}$ & $1.31_{-1.31}^{+0.11}$ & $0.69_{-0.48}^{+0.46}$ & $3.4_{-1.4}^{+1.6}$ & 0 & 2 stars in aper; possible blended EB \\
\hline $\mathrm{C} 7$ & 214741009.01 & 14.0 & 0.73 & $P$ & 7.3 & $41.56_{-27.99}^{+38.08}$ & $2.36_{-0.15}^{+0.14}$ & $1.29_{-0.34}^{+0.40}$ & $32.9_{-25.8}^{+32.9}$ & 0 & V-shaped \\
\hline C7 & 217149884.01 & 14.2 & 0.90 & $\mathrm{P}$ & 16.7 & $17.33_{-0.13}^{+0.18}$ & $5.53_{-0.04}^{+0.04}$ & $0.54_{-0.03}^{+0.02}$ & $17.0_{-6.8}^{+6.8}$ & 0 & Deep $(\sim 3 \%)$; flat-bottomed; \\
\hline C7 & 215969174.01 & 14.3 & 1.15 & S & 4.2 & $10.69_{-0.04}^{+0.07}$ & $3.38_{-0.01}^{+0.01}$ & $0.11_{-0.07}^{+0.10}$ & $13.4_{-1.1}^{+1.5}$ & 0 & $\ldots$ \\
\hline C7 & 215101303.01 & 14.9 & 0.84 & $\mathrm{P}$ & 15.2 & $13.85_{-0.20}^{+0.40}$ & $3.55_{-0.05}^{+0.07}$ & $0.28_{-0.19}^{+0.19}$ & $12.7_{-5.1}^{+5.1}$ & 0 & Deep ( $3 \%$ ); flat-bottomed; \\
\hline C7 & 213951550.01 & 15.0 & 0.27 & $P$ & 1.1 & $57.27_{-18.47}^{+26.37}$ & $1.78_{-0.08}^{+0.09}$ & $1.11_{-0.27}^{+0.32}$ & $16.9_{-8.7}^{+10.3}$ & 0 & Deep ( 6\%); V-shaped; \\
\hline C7 & 217393088.01 & 15.3 & 1.54 & S & 1.3 & $9.87_{-0.08}^{+0.15}$ & $3.13_{-0.03}^{+0.03}$ & $0.16_{-0.11}^{+0.15}$ & $16.6_{-2.0}^{+2.4}$ & 0 & $\ldots$ \\
\hline $\mathrm{C} 8$ & 220542353.01 & 8.8 & 1.91 & S & 15.2 & $37.44_{-5.31}^{+9.47}$ & $3.76_{-0.01}^{+0.01}$ & $1.06_{-0.07}^{+0.11}$ & $78.0_{-15.1}^{+23.2}$ & 0 & Deep ( 4\%); V-shaped \\
\hline $\mathrm{C} 8$ & 220383386.01 & 8.9 & 0.88 & S & 1.0 & $1.83_{-0.09}^{+0.22}$ & $1.65_{-0.08}^{+0.05}$ & $0.49_{-0.33}^{+0.33}$ & $1.8_{-0.1}^{+0.2}$ & 0 & Multi;HD3167b; Vanderburg +16 \\
\hline $\mathrm{C} 8$ & 220383386.02 & 8.9 & 0.88 & S & 29.8 & $3.12_{-0.24}^{+0.51}$ & $5.06_{-0.17}^{+0.49}$ & $0.63_{-0.42}^{+0.27}$ & $3.0_{-0.3}^{+0.5}$ & 0 & Multi;HD3167c; Vanderburg +16 \\
\hline $\mathrm{C} 8$ & 220666988.01 & 9.3 & 1.03 & $\mathrm{P}$ & 0.9 & $37.34_{-21.21}^{+28.78}$ & $0.00_{-0.00}^{+1.11}$ & $1.50_{-0.38}^{+0.45}$ & $42.0_{-29.2}^{+36.5}$ & 1 & $\ldots$ \\
\hline $\mathrm{C} 8$ & 220709978.01 & 9.4 & 0.96 & S & 15.4 & $2.02_{-0.10}^{+0.22}$ & $4.39_{-0.16}^{+0.17}$ & $0.47_{-0.32}^{+0.30}$ & $2.1_{-0.2}^{+0.3}$ & 0 & $\ldots$ \\
\hline $\mathrm{C} 8$ & 220303276.01 & 10.9 & 1.38 & S & 4.0 & $8.03_{-0.01}^{+0.01}$ & $4.93_{-0.01}^{+0.01}$ & $0.04_{-0.03}^{+0.04}$ & $12.0_{-0.9}^{+1.2}$ & 0 & WASP-118b \\
\hline $\mathrm{C} 8$ & 220725183.01 & 11.5 & 1.58 & S & 2.3 & $30.02_{-0.60}^{+0.72}$ & $4.03_{-0.00}^{+0.00}$ & $0.88_{-0.01}^{+0.01}$ & $51.6_{-6.2}^{+7.6}$ & 0 & Deep ( $\sim 5 \%)$; V-shape \\
\hline $\mathrm{C} 8$ & 220376054.01 & 11.6 & 1.29 & $\mathrm{~S}$ & 8.6 & $1.75_{-0.09}^{+0.22}$ & $3.73_{-0.12}^{+0.14}$ & $0.48_{-0.33}^{+0.31}$ & $2.5_{-0.3}^{+0.5}$ & 0 & $\ldots$ \\
\hline $\mathrm{C} 8$ & 220621788.01 & 11.8 & 0.99 & $\mathrm{~S}$ & 13.7 & $2.13_{-0.11}^{+0.25}$ & $3.12_{-0.10}^{+0.11}$ & $0.47_{-0.32}^{+0.30}$ & $2.3_{-0.2}^{+0.4}$ & 0 & $\ldots$ \\
\hline $\mathrm{C} 8$ & 220674823.01 & 12.0 & 0.97 & $\mathrm{~S}$ & 0.6 & $1.74_{-0.08}^{+0.19}$ & $1.45_{-0.16}^{+0.07}$ & $0.48_{-0.33}^{+0.38}$ & $1.8_{-0.1}^{+0.3}$ & 0 & Multi \\
\hline $\mathrm{C} 8$ & 220674823.02 & 12.0 & 0.97 & $\mathrm{~S}$ & 13.3 & $2.65_{-0.15}^{+0.36}$ & $3.44_{-0.16}^{+0.18}$ & $0.47_{-0.32}^{+0.32}$ & $2.8_{-0.2}^{+0.5}$ & 0 & Multi \\
\hline $\mathrm{C} 8$ & 220481411.01 & 12.1 & 0.71 & $\mathrm{~S}$ & 2.2 & $2.22_{-0.12}^{+0.29}$ & $1.83_{-0.05}^{+0.05}$ & $0.49_{-0.33}^{+0.31}$ & $1.7_{-0.3}^{+0.3}$ & 0 & $\cdots$ \\
\hline $\mathrm{C} 8$ & 220294712.01 & 12.3 & 1.09 & $\mathrm{~S}$ & 23.6 & $2.51_{-0.10}^{+0.24}$ & $5.84_{-0.17}^{+0.20}$ & $0.46_{-0.31}^{+0.29}$ & $3.0_{-0.3}^{+0.4}$ & 0 & $\ldots$ \\
\hline $\mathrm{C} 8$ & 220555384.01 & 12.4 & 0.55 & $P$ & 4.3 & $2.01_{-0.15}^{+0.34}$ & $1.17_{-0.08}^{+0.08}$ & $0.50_{-0.34}^{+0.31}$ & $1.2_{-0.5}^{+0.5}$ & 0 & V-shaped \\
\hline $\mathrm{C} 8$ & 220321605.01 & 12.6 & 0.67 & $\mathrm{~S}$ & 9.8 & $3.57_{-0.18}^{+0.40}$ & $2.60_{-0.05}^{+0.07}$ & $0.45_{-0.29}^{+0.26}$ & $2.6_{-0.4}^{+0.5}$ & 0 & $\cdots$ \\
\hline $\mathrm{C} 8$ & 220397060.01 & 12.8 & 0.84 & $\mathrm{~S}$ & 12.1 & $5.10_{-0.11}^{+0.29}$ & $8.51_{-0.08}^{+0.11}$ & $0.28_{-0.19}^{+0.22}$ & $4.7_{-0.4}^{+5.1}$ & 0 & $\cdots$ \\
\hline $\mathrm{C} 8$ & 220187552.01 & 12.8 & 0.66 & $\mathrm{~S}$ & 17.1 & $45.14_{-18.70}^{+22.57}$ & $2.26_{-0.03}^{+0.02}$ & $1.20_{-0.23}^{+0.31}$ & $32.5_{-14.3}^{+21.2}$ & 0 & Deep ( 2\%); V-shaped \\
\hline $\mathrm{C} 8$ & 220431824.01 & 13.0 & 1.45 & $\mathrm{~S}$ & 9.1 & $11.55_{-0.02}^{+0.04}$ & $6.91_{-0.01}^{+0.02}$ & $0.07_{-0.05}^{+0.07}$ & $18.3_{-3.1}^{+2.4}$ & 1 & $\cdots$ \\
\hline $\mathrm{C} 8$ & 220621087.01 & 13.4 & 0.45 & $\mathrm{~S}$ & 3.8 & $2.99_{-0.24}^{+0.53}$ & $1.54_{-0.10}^{+0.11}$ & $0.52_{-0.35}^{+0.29}$ & $1.5_{-0.3}^{+0.4}$ & 0 & $\cdots$ \\
\hline $\mathrm{C} 8$ & 220504338.01 & 13.5 & 1.33 & $\mathrm{~S}$ & 5.8 & $8.65_{-0.25}^{+0.21}$ & $2.89_{-0.06}^{+0.06}$ & $0.58_{-0.15}^{+0.08}$ & $12.5_{-1.6}^{+1.7}$ & 0 & $\cdots$ \\
\hline $\mathrm{C} 8$ & 220501947.01 & 13.5 & 0.73 & $\mathrm{~S}$ & 4.0 & $12.94_{-0.02}^{+0.04}$ & $2.48_{-0.00}^{+0.01}$ & $0.06_{-0.04}^{+0.06}$ & $10.3_{-1.4}^{+1.4}$ & 0 & Deep $(\sim 2 \%)$; V-shape \\
\hline $\mathrm{C} 8$ & 220258394.01 & 13.7 & 0.87 & $\mathrm{~S}$ & 16.0 & $20.49_{-0.10}^{+0.13}$ & $4.89_{-0.02}^{+0.02}$ & $0.70_{-0.01}^{+0.01}$ & $19.5_{-0.8}^{+0.9}$ & 0 & Deep ( 5\%); V-shape \\
\hline $\mathrm{C} 8$ & 220554210.01 & 13.7 & 0.93 & S & 4.2 & $2.70_{-0.14}^{+0.34}$ & $2.73_{-0.11}^{+0.11}$ & $0.48_{-0.32}^{+0.31}$ & $2.7_{-0.2}^{+0.4}$ & 0 & $\ldots$ \\
\hline $\mathrm{C} 8$ & 220436208.01 & 13.9 & 1.17 & $\mathrm{~S}$ & 5.2 & $3.37_{-0.13}^{+0.34}$ & $3.45_{-0.09}^{+0.10}$ & $0.44_{-0.30}^{+0.30}$ & $4.3_{-0.5}^{+0.7}$ & 0 & $\cdots$ \\
\hline $\mathrm{C} 8$ & 220629489.01 & 14.1 & 0.86 & $\mathrm{~S}$ & 1.9 & $4.04_{-0.18}^{+0.48}$ & $1.75_{-0.04}^{+0.06}$ & $0.45_{-0.31}^{+0.32}$ & $3.8_{-0.2}^{+0.5}$ & 0 & $\cdots$ \\
\hline $\mathrm{C} 8$ & 220565349.01 & 14.1 & 0.84 & $\mathrm{~S}$ & 21.8 & $21.77_{-6.56}^{+23.83}$ & $2.20_{-0.21}^{+0.05}$ & $0.91_{-0.23}^{+0.31}$ & $19.9_{-6.0}^{+21.8}$ & 0 & Deep ( 2\%); V-shape \\
\hline $\mathrm{C} 8$ & 220209578.01 & 14.4 & 0.89 & $\mathrm{~S}$ & 8.9 & $38.05_{-20.38}^{+32.87}$ & $2.95_{-0.07}^{+0.06}$ & $1.24_{-0.25}^{+0.35}$ & $36.9_{-19.9}^{+32.0}$ & 0 & V-shaped \\
\hline $\mathrm{C} 8$ & 220522262.01 & 14.8 & 0.75 & $\mathrm{~S}$ & 8.7 & $9.89_{-0.14}^{+0.33}$ & $2.75_{-0.03}^{+0.04}$ & $0.23_{-0.16}^{+0.21}$ & $8.1_{-1.1}^{+1.1}$ & 0 & $\cdots$ \\
\hline $\mathrm{C} 8$ & 220696233.01 & 15.5 & 0.35 & $\mathrm{P}$ & 28.7 & $10.49_{-0.38}^{+0.88}$ & $2.99_{-0.11}^{+0.15}$ & $0.36_{-0.25}^{+0.26}$ & $4.0_{-1.6}^{+1.6}$ & 0 & $\ldots$ \\
\hline $\mathrm{C} 8$ & 220336320.01 & 15.9 & 0.37 & $\mathrm{P}$ & 1.7 & $52.53_{-21.65}^{+25.35}$ & $1.37_{-0.02}^{+0.02}$ & $1.17_{-0.29}^{+0.29}$ & $21.3_{-12.2}^{+13.3}$ & 0 & Deep ( 5\%); V-shaped \\
\hline $\mathrm{C} 8$ & 220448185.01 & 16.0 & 0.69 & $\mathrm{P}$ & 0.7 & $22.87_{-3.88}^{+42.13}$ & $0.37_{-0.06}^{+0.07}$ & $0.67_{-0.49}^{+0.66}$ & $17.2_{-7.5}^{+32.5}$ & 0 & Half the reported period \\
\hline
\end{tabular}

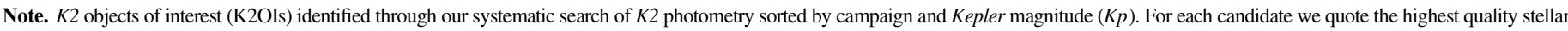

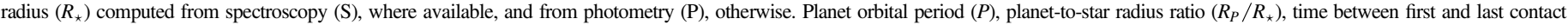

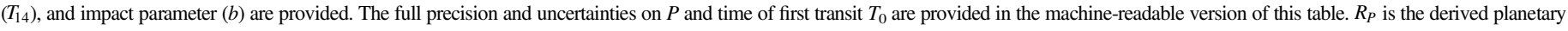

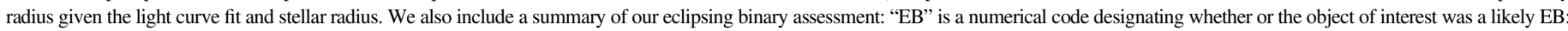
0 - no obvious indication of EB, 1 - secondary eclipse visible, 2-photometric variability that is phase-locked to the eclipse. Some additional comments on individual systems are provided.

(This table is available in machine-readable form.)

In our modeling, the following parameters are allowed to vary: time of first transit $T_{0}$, orbital period $P$, inclination $i$, scaled semimajor axis $a / R_{\star}$, planet-star radius ratio $R_{P} / R_{\star}$, orbital eccentricity $e$, longitude of periastron $\omega$, linear limbdarkening coefficient $u$, fractional light curve dilution $\delta$, and the out-of-transit flux level.

During the fitting, we adopted the following priors.

\section{Period. Gaussian prior centered on maximum likelihood $P$ having dispersion of 0.01 days. ${ }^{19}$}

\footnotetext{
${ }^{19}$ We imposed weak priors on $P$ and $T_{0}$ to keep the MCMC walkers from jumping too far from the likelihood mode and wandering away. After performing the MCMC exploration, we verified that $P$ and $T_{0}$ were more tightly constrained by the photometry than by the priors. The uncertainties on $T_{0}$ are typically $2.5 \%$ of the prior width (median value) and no more than $50 \%$ the prior width. The uncertainties on P are typically $2 \%$ of the prior width (median value) and no more than $60 \%$ the prior width.
}

2. Time of transit. Uniform prior centered on maximum likelihood $T_{0}$ having dispersion of $0.06 \times P$.

3. Radius ratio. Uniform prior, $R_{P} / R_{\star}=[-1,+1]$. Following Eastman et al. (2013), we allow for negative $R_{P} / R_{\star}$ in our sampling to avoid the Lucy-Sweeney-type bias that results from treating $R_{P} / R_{\star}$ as a positive-definite quantity (Lucy \& Sweeney 1971).

4. Eccentricity. Gaussian prior centered at $10^{-4}$ having dispersion of $10^{-3}$. This effectively restricts the orbits to circular.

5. Longitude of periastron. Uniform prior, $\omega=[0,2 \pi]$.

6. Inclination. Uniform prior, $i=\left[50^{\circ}, 90^{\circ}\right]$.

7. Limb-darkening. Gaussian prior on $u$ where the mean and dispersion are computed using the publicly available Limb-darkening Toolkit (LDTK; Parviainen \& Aigrain 2015). LDTK computes the distribution of $u$ given 
Table 2

Stars with HIRES Spectra and Derived Parameters

\begin{tabular}{|c|c|c|c|c|c|c|c|c|c|c|c|}
\hline Camp. & EPIC & $\begin{array}{c}K p \\
(\mathrm{mag})\end{array}$ & $\begin{array}{l}T_{\text {eff }} \\
(\mathrm{K})\end{array}$ & $\begin{array}{l}\log g \\
(\mathrm{cgs})\end{array}$ & $\begin{array}{c}{[\mathrm{Fe} / \mathrm{H}]} \\
(\mathrm{dex})\end{array}$ & $\begin{array}{c}v \sin i \\
\left(\mathrm{~km} \mathrm{~s}^{-1}\right)\end{array}$ & $\begin{array}{l}M_{\star} \\
M_{\odot}\end{array}$ & $\begin{array}{l}R_{\star} \\
R_{\odot}\end{array}$ & $\mathrm{SM}^{\mathrm{a}}$ & $\mathrm{SB} 2^{\mathrm{b}}$ & $\operatorname{disp}^{c}$ \\
\hline $\mathrm{C} 5$ & 211401787 & 9.7 & 6214 & 4.26 & -0.04 & 8.4 & $1.12_{-0.05}^{+0.06}$ & $1.25_{-0.12}^{+0.17}$ & 1 & 1 & $\mathrm{PC}$ \\
\hline $\mathrm{C} 5$ & 211945201 & 10.1 & 6018 & 4.13 & 0.12 & 3.3 & $1.17_{-0.08}^{+0.10}$ & $1.48_{-0.19}^{+0.21}$ & 1 & 1 & PC \\
\hline $\mathrm{C} 5$ & 211990866 & 10.4 & 6180 & 4.51 & 0.32 & 13.6 & $1.25_{-0.04}^{+0.05}$ & $1.23_{-0.06}^{+0.09}$ & 1 & 1 & $\mathrm{PC}$ \\
\hline C5 & 212099230 & 10.5 & 5487 & 4.41 & 0.11 & 1.6 & $0.93_{-0.04}^{+0.04}$ & $0.96_{-0.06}^{+0.09}$ & 1 & 1 & $\mathrm{PC}$ \\
\hline $\mathrm{C} 5$ & 211594205 & 10.7 & 5240 & 4.69 & -0.05 & 1.5 & $0.83_{-0.03}^{+0.03}$ & $0.79_{-0.03}^{+0.04}$ & 1 & 1 & PC \\
\hline $\mathrm{C} 5$ & 212110888 & 11.4 & 6008 & 4.16 & 0.01 & 5.9 & $1.09_{-0.06}^{+0.09}$ & $1.38_{-0.17}^{+0.19}$ & 1 & 4 & PC \\
\hline $\mathrm{C} 5$ & 211525389 & 11.7 & 5479 & 4.48 & 0.30 & 2.4 & $1.00_{-0.04}^{+0.04}$ & $0.98_{-0.05}^{+0.08}$ & 1 & 1 & PC \\
\hline $\mathrm{C} 5$ & 211359660 & 11.7 & 5177 & 4.62 & 0.12 & 2.0 & $0.87_{-0.03}^{+0.03}$ & $0.82_{-0.03}^{+0.04}$ & 1 & 1 & $\mathrm{PC}$ \\
\hline $\mathrm{C} 5$ & 212012119 & 11.8 & 4929 & 4.73 & 0.05 & 4.7 & $0.80_{-0.03}^{+0.03}$ & $0.75_{-0.02}^{+0.03}$ & 1 & 1 & $\mathrm{PC}$ \\
\hline $\mathrm{C} 5$ & 211491383 & 11.8 & 6141 & 4.21 & -0.13 & 6.1 & $1.06_{-0.05}^{+0.06}$ & $1.28_{-0.15}^{+0.18}$ & 1 & 1 & $\mathrm{PC}$ \\
\hline $\mathrm{C} 5$ & 211391664 & 12.1 & 6074 & 4.12 & -0.11 & 6.7 & $1.05_{-0.06}^{+0.08}$ & $1.39_{-0.17}^{+0.19}$ & 1 & 1 & $\mathrm{PC}$ \\
\hline $\mathrm{C} 5$ & 211736671 & 12.2 & 5554 & 3.93 & 0.40 & 2.6 & $1.18_{-0.09}^{+0.10}$ & $1.77_{-0.23}^{+0.27}$ & 1 & 1 & $\mathrm{PC}$ \\
\hline $\mathrm{C} 5$ & 211319617 & 12.4 & 5156 & 4.72 & -0.60 & 0.8 & $0.69_{-0.03}^{+0.03}$ & $0.65_{-0.02}^{+0.02}$ & 1 & 1 & $\mathrm{PC}$ \\
\hline $\mathrm{C} 5$ & 211342524 & 12.4 & 6168 & 4.14 & -0.19 & 7.8 & $1.04_{-0.06}^{+0.07}$ & $1.36_{-0.16}^{+0.19}$ & 1 & 5 & $\mathrm{PC}$ \\
\hline $\mathrm{C} 5$ & 211351816 & 12.4 & 4803 & 3.19 & 0.40 & 3.7 & $1.45_{-0.22}^{+0.25}$ & $4.79_{-0.67}^{+0.86}$ & 1 & 1 & PC \\
\hline $\mathrm{C} 5$ & 211800191 & 12.4 & 5919 & 4.53 & -0.51 & 1.3 & $0.85_{-0.04}^{+0.04}$ & $0.84_{-0.04}^{+0.06}$ & 1 & 1 & $\mathrm{PC}$ \\
\hline $\mathrm{C} 5$ & 212006344 & 12.5 & 3925 & $\ldots$ & 0.43 & $\ldots$ & .. & $0.63_{-0.10}^{+0.10}$ & 2 & 1 & $\mathrm{PC}$ \\
\hline $\mathrm{C} 5$ & 211355342 & 12.6 & 5606 & 4.32 & 0.27 & 1.9 & $1.03_{-0.05}^{+0.05}$ & $1.11_{-0.10}^{+0.14}$ & 1 & 1 & PC \\
\hline $\mathrm{C} 5$ & 212164470 & 12.7 & 5893 & 4.24 & -0.04 & 2.8 & $1.00_{-0.05}^{+0.05}$ & $1.19_{-0.13}^{+0.16}$ & 1 & 1 & $\mathrm{PC}$ \\
\hline $\mathrm{C} 5$ & 211562654 & 12.8 & 5442 & 4.43 & 0.09 & 1.2 & $0.91_{-0.04}^{+0.04}$ & $0.92_{-0.06}^{+0.08}$ & 1 & 1 & $\mathrm{PC}$ \\
\hline $\mathrm{C} 5$ & 212008766 & 12.8 & 5038 & 4.68 & -0.12 & 1.7 & $0.77_{-0.03}^{+0.03}$ & $0.74_{-0.03}^{+0.03}$ & 1 & 1 & $\mathrm{PC}$ \\
\hline $\mathrm{C} 5$ & 212157262 & 12.9 & 5459 & 4.48 & 0.25 & 1.6 & $0.97_{-0.04}^{+0.04}$ & $0.96_{-0.05}^{+0.07}$ & 1 & 1 & $\mathrm{PC}$ \\
\hline $\mathrm{C} 5$ & 212138198 & 12.9 & 5138 & 4.40 & 0.29 & 1.9 & $0.90_{-0.03}^{+0.03}$ & $0.89_{-0.05}^{+0.06}$ & 1 & 1 & PC \\
\hline $\mathrm{C} 5$ & 211818569 & 12.9 & 4526 & $\ldots$ & 0.04 & $\ldots$ & $\ldots$ & $0.71_{-0.10}^{+0.10}$ & 2 & 1 & $\mathrm{PC}$ \\
\hline $\mathrm{C} 5$ & 211439059 & 13.1 & 5481 & 4.72 & -0.01 & 4.3 & $0.90_{-0.04}^{+0.03}$ & $0.84_{-0.03}^{+0.04}$ & 1 & 4 & $\mathrm{PC}$ \\
\hline $\mathrm{C} 5$ & 211919004 & 13.1 & 5149 & 4.51 & 0.22 & 1.4 & $0.89_{-0.03}^{+0.04}$ & $0.86_{-0.04}^{+0.05}$ & 1 & 1 & $\mathrm{PC}$ \\
\hline $\mathrm{C} 5$ & 211442297 & 13.2 & 5596 & 4.55 & -0.12 & 0.9 & $0.89_{-0.04}^{+0.04}$ & $0.86_{-0.04}^{+0.06}$ & 1 & 1 & $\mathrm{PC}$ \\
\hline $\mathrm{C} 5$ & 211490999 & 13.4 & 5488 & 4.42 & -0.02 & 0.9 & $0.89_{-0.04}^{+0.04}$ & $0.91_{-0.06}^{+0.07}$ & 1 & 1 & PC \\
\hline $\mathrm{C} 5$ & 211529065 & 13.4 & 4915 & 4.53 & 0.31 & 3.5 & $0.86_{-0.03}^{+0.03}$ & $0.82_{-0.03}^{+0.03}$ & 1 & 1 & $\mathrm{PC}$ \\
\hline $\mathrm{C} 5$ & 211413752 & 13.5 & 5025 & 4.61 & 0.04 & 1.5 & $0.81_{-0.03}^{+0.03}$ & $0.78_{-0.03}^{+0.03}$ & 1 & 1 & $\mathrm{PC}$ \\
\hline $\mathrm{C} 5$ & 211713099 & 13.6 & 5532 & 4.37 & -0.35 & 0.6 & $0.80_{-0.03}^{+0.03}$ & $0.83_{-0.05}^{+0.06}$ & 1 & 1 & $\mathrm{PC}$ \\
\hline $\mathrm{C} 5$ & 211816003 & 13.7 & 5313 & 4.61 & -0.34 & 0.3 & $0.77_{-0.03}^{+0.03}$ & $0.74_{-0.03}^{+0.03}$ & 1 & 1 & $\mathrm{PC}$ \\
\hline $\mathrm{C} 5$ & 211331236 & 13.9 & 3687 & $\ldots$ & -0.17 & $\ldots$ & $\ldots$ & $0.52_{-0.10}^{+0.10}$ & 2 & 1 & $\mathrm{PC}$ \\
\hline $\mathrm{C} 5$ & 212069861 & 14.1 & 3926 & $\ldots$ & 0.18 & $\ldots$ & $\ldots$ & $0.64_{-0.10}^{+0.10}$ & 2 & 1 & $\mathrm{PC}$ \\
\hline $\mathrm{C} 5$ & 211929937 & 14.2 & 5230 & 4.54 & 0.10 & 0.6 & $0.87_{-0.03}^{+0.03}$ & $0.84_{-0.04}^{+0.04}$ & 1 & 1 & $\mathrm{PC}$ \\
\hline $\mathrm{C} 5$ & 211418729 & 14.3 & 5020 & 4.33 & 0.45 & 2.7 & $0.90_{-0.03}^{+0.03}$ & $0.90_{-0.05}^{+0.06}$ & 1 & 1 & $\mathrm{PC}$ \\
\hline $\mathrm{C} 5$ & 211578235 & 14.3 & 5491 & 4.25 & -0.16 & 4.1 & $0.85_{-0.03}^{+0.04}$ & $0.94_{-0.08}^{+0.14}$ & 1 & 5 & $\mathrm{PC}$ \\
\hline $\mathrm{C} 5$ & 211399359 & 14.4 & 4972 & 4.64 & 0.04 & 3.2 & $0.80_{-0.03}^{+0.03}$ & $0.77_{-0.03}^{+0.03}$ & 1 & 1 & $\mathrm{PC}$ \\
\hline $\mathrm{C} 5$ & 211978865 & 14.4 & 6519 & 4.33 & -0.19 & 18.7 & $1.15_{-0.05}^{+0.05}$ & $1.21_{-0.08}^{+0.13}$ & 1 & 3 & $\mathrm{PC}$ \\
\hline $\mathrm{C} 5$ & 212130773 & 14.5 & 4949 & 4.55 & 0.04 & 1.8 & $0.80_{-0.03}^{+0.03}$ & $0.77_{-0.03}^{+0.03}$ & 1 & 1 & $\mathrm{PC}$ \\
\hline C5 & 211770795 & 14.5 & 4454 & $\ldots$ & 0.08 & $\ldots$ & $\ldots$ & $0.71_{-0.10}^{+0.10}$ & 2 & 1 & $\mathrm{PC}$ \\
\hline $\mathrm{C} 5$ & 212154564 & 15.1 & $\ldots$ & $\ldots$ & $\ldots$ & 65.7 & $\ldots$ & $\ldots$ & 0 & 1 & $\mathrm{PC}$ \\
\hline $\mathrm{C} 5$ & 211916756 & 15.5 & $\ldots$ & $\ldots$ & $\ldots$ & 252.7 & $\ldots$ & $\ldots$ & 0 & 1 & PC \\
\hline C6 & 212357477 & 10.2 & 5741 & 4.46 & 0.12 & 2.4 & $1.02_{-0.05}^{+0.05}$ & $1.01_{-0.06}^{+0.08}$ & 1 & 1 & $\mathrm{PC}$ \\
\hline C6 & 212703473 & 10.7 & 5816 & 4.38 & 0.19 & 5.2 & $1.08_{-0.05}^{+0.05}$ & $1.11_{-0.08}^{+0.12}$ & 1 & 3 & $\mathrm{PC}$ \\
\hline C6 & 212803289 & 11.0 & 6102 & 3.96 & 0.20 & 10.0 & $1.40_{-0.12}^{+0.13}$ & $1.98_{-0.27}^{+0.31}$ & 1 & 1 & $\mathrm{PC}$ \\
\hline C6 & 212782836 & 11.6 & 5418 & 4.48 & -0.42 & 1.1 & $0.76_{-0.03}^{+0.03}$ & $0.76_{-0.03}^{+0.04}$ & 1 & 1 & $\mathrm{PC}$ \\
\hline C6 & 212521166 & 11.6 & 4895 & 4.64 & -0.24 & 1.9 & $0.71_{-0.03}^{+0.03}$ & $0.68_{-0.02}^{+0.02}$ & 1 & 1 & $\mathrm{PC}$ \\
\hline C6 & 212586030 & 11.7 & 4865 & 3.37 & 0.38 & 3.5 & $1.41_{-0.19}^{+0.21}$ & $3.83_{-0.52}^{+0.62}$ & 1 & 1 & $\mathrm{PC}$ \\
\hline C6 & 212300977 & 11.7 & 5965 & 4.34 & 0.00 & 2.7 & $1.04_{-0.05}^{+0.05}$ & $1.11_{-0.09}^{+0.13}$ & 1 & 1 & $\mathrm{PC}$ \\
\hline C6 & 212779596 & 11.9 & 4507 & $\ldots$ & -0.04 & $\ldots$ & $\ldots$ & $0.70_{-0.10}^{+0.10}$ & 2 & 1 & $\mathrm{PC}$ \\
\hline C6 & 212735333 & 12.0 & 5660 & 4.50 & 0.09 & 1.3 & $0.98_{-0.05}^{+0.04}$ & $0.96_{-0.05}^{+0.07}$ & 1 & 1 & $\mathrm{PC}$ \\
\hline C6 & 212587672 & 12.2 & 5948 & 4.49 & -0.21 & 2.1 & $0.95_{-0.04}^{+0.04}$ & $0.95_{-0.05}^{+0.08}$ & 1 & 1 & PC \\
\hline C6 & 212697709 & 12.2 & 5719 & 4.28 & 0.28 & 1.6 & $1.09_{-0.05}^{+0.06}$ & $1.20_{-0.13}^{+0.17}$ & 1 & 1 & $\mathrm{PC}$ \\
\hline C6 & 212394689 & 12.2 & 5456 & 4.50 & -0.01 & 1.6 & $0.89_{-0.04}^{+0.04}$ & $0.88_{-0.04}^{+0.06}$ & 1 & 1 & $\mathrm{PC}$ \\
\hline C6 & 212689874 & 12.3 & 5644 & 4.36 & -0.12 & 1.7 & $0.88_{-0.04}^{+0.04}$ & $0.95_{-0.07}^{+0.10}$ & 1 & 1 & $\mathrm{PC}$ \\
\hline C6 & 212435047 & 12.4 & 5750 & 4.29 & 0.01 & 2.0 & $0.96_{-0.05}^{+0.05}$ & $1.10_{-0.11}^{+0.14}$ & 1 & 1 & $\mathrm{PC}$ \\
\hline
\end{tabular}


Table 2

(Continued)

\begin{tabular}{|c|c|c|c|c|c|c|c|c|c|c|c|}
\hline Camp. & EPIC & $\begin{array}{c}K p \\
(\mathrm{mag})\end{array}$ & $\begin{array}{l}T_{\text {eff }} \\
(\mathrm{K})\end{array}$ & $\begin{array}{l}\log g \\
\text { (cgs) }\end{array}$ & $\begin{array}{c}{[\mathrm{Fe} / \mathrm{H}]} \\
(\mathrm{dex})\end{array}$ & $\begin{array}{c}v \sin i \\
\left(\mathrm{~km} \mathrm{~s}^{-1}\right)\end{array}$ & $\begin{array}{l}M_{\star} \\
M_{\odot}\end{array}$ & $\begin{array}{l}R_{\star} \\
R_{\odot}\end{array}$ & $\mathrm{SM}^{\mathrm{a}}$ & $\mathrm{SB} 2^{\mathrm{b}}$ & $\operatorname{disp}^{c}$ \\
\hline C6 & 212460519 & 12.4 & 4226 & $\ldots$ & -0.17 & $\ldots$ & $\ldots$ & $0.66_{-0.10}^{+0.10}$ & 2 & 1 & $\mathrm{PC}$ \\
\hline $\mathrm{C} 6$ & 212428509 & 12.5 & 5697 & 4.25 & -0.42 & 1.7 & $0.81_{-0.03}^{+0.03}$ & $0.94_{-0.08}^{+0.15}$ & 1 & 1 & PC \\
\hline C6 & 212585579 & 12.6 & 5931 & 4.35 & -0.00 & 2.3 & $1.02_{-0.05}^{+0.05}$ & $1.09_{-0.09}^{+0.12}$ & 1 & 1 & $\mathrm{PC}$ \\
\hline $\mathrm{C} 7$ & 213920015 & 10.0 & 5682 & 4.60 & -0.12 & 2.1 & $0.91_{-0.04}^{+0.04}$ & $0.87_{-0.04}^{+0.05}$ & 1 & 1 & PC \\
\hline $\mathrm{C} 7$ & 218711655 & 11.3 & 6404 & 4.18 & 0.08 & 6.9 & $1.29_{-0.06}^{+0.08}$ & $1.46_{-0.16}^{+0.22}$ & 1 & 1 & $\mathrm{PC}$ \\
\hline $\mathrm{C} 7$ & 218916923 & 11.5 & 5393 & 4.57 & 0.29 & 1.7 & $0.97_{-0.04}^{+0.04}$ & $0.93_{-0.04}^{+0.06}$ & 1 & 1 & $\mathrm{PC}$ \\
\hline $\mathrm{C} 7$ & 214611894 & 11.9 & 6072 & 4.27 & -0.02 & 4.8 & $1.07_{-0.05}^{+0.06}$ & $1.21_{-0.12}^{+0.17}$ & 1 & 1 & PC \\
\hline $\mathrm{C} 7$ & 213546283 & 12.0 & 5685 & 4.23 & -0.14 & 0.1 & $0.89_{-0.03}^{+0.04}$ & $1.10_{-0.12}^{+0.17}$ & 1 & 1 & $\mathrm{PC}$ \\
\hline C7 & 215938010 & 12.1 & 6027 & 4.03 & 0.08 & 3.5 & $1.20_{-0.09}^{+0.11}$ & $1.64_{-0.20}^{+0.24}$ & 1 & 1 & $\mathrm{PC}$ \\
\hline $\mathrm{C} 7$ & 216494238 & 12.3 & 5741 & 4.14 & 0.35 & 2.5 & $1.17_{-0.08}^{+0.10}$ & $1.45_{-0.18}^{+0.20}$ & 1 & 1 & PC \\
\hline $\mathrm{C} 7$ & 219388192 & 12.3 & 5781 & 4.54 & 0.12 & 4.2 & $1.03_{-0.04}^{+0.05}$ & $1.00_{-0.05}^{+0.07}$ & 1 & 1 & $\mathrm{PC}$ \\
\hline $\mathrm{C} 7$ & 218621322 & 12.4 & 5675 & 4.26 & -0.27 & 0.3 & $0.85_{-0.03}^{+0.03}$ & $1.00_{-0.10}^{+0.16}$ & 1 & 1 & PC \\
\hline $\mathrm{C} 7$ & 216892056 & 12.5 & $\ldots$ & $\ldots$ & $\ldots$ & 64.1 & $\ldots$ & $\ldots$ & 0 & 1 & PC \\
\hline $\mathrm{C} 7$ & 217192839 & 12.6 & 4541 & $\ldots$ & -0.28 & $\ldots$ & $\ldots$ & $0.70_{-0.10}^{+0.10}$ & 2 & 1 & PC \\
\hline $\mathrm{C} 7$ & 218131080 & 12.7 & 6394 & 4.32 & -0.09 & 4.5 & $1.15_{-0.05}^{+0.05}$ & $1.22_{-0.09}^{+0.14}$ & 1 & 1 & $\mathrm{PC}$ \\
\hline $\mathrm{C} 7$ & 216468514 & 12.7 & 6038 & 4.02 & 0.16 & 4.7 & $1.28_{-0.11}^{+0.12}$ & $1.73_{-0.23}^{+0.26}$ & 1 & 1 & PC \\
\hline $\mathrm{C} 7$ & 219420915 & 12.8 & 5815 & 4.24 & 0.32 & 2.9 & $1.15_{-0.06}^{+0.08}$ & $1.30_{-0.14}^{+0.18}$ & 1 & 1 & PC \\
\hline $\mathrm{C} 7$ & 216334329 & 12.9 & 5830 & 4.05 & 0.13 & 2.3 & $1.12_{-0.07}^{+0.10}$ & $1.54_{-0.18}^{+0.23}$ & 1 & 1 & PC \\
\hline $\mathrm{C} 7$ & 219256848 & 13.1 & 4530 & 2.57 & 0.32 & 4.2 & $1.76_{-0.35}^{+0.44}$ & $11.57_{-0.94}^{+1.03}$ & 1 & 1 & $\mathrm{PC}$ \\
\hline $\mathrm{C} 7$ & 217671466 & 13.1 & 5576 & 3.87 & 0.43 & 3.7 & $1.25_{-0.10}^{+0.12}$ & $1.94_{-0.25}^{+0.33}$ & 1 & 1 & PC \\
\hline $\mathrm{C} 7$ & 215389654 & 13.2 & 5416 & 4.41 & 0.24 & 1.2 & $0.96_{-0.04}^{+0.04}$ & $0.97_{-0.06}^{+0.09}$ & 1 & 1 & PC \\
\hline $\mathrm{C} 7$ & 213840781 & 13.7 & $\ldots$ & $\ldots$ & $\ldots$ & 137.1 & $\ldots$ & $\ldots$ & 0 & 5 & PC \\
\hline $\mathrm{C} 7$ & 215358983 & 13.8 & 6135 & 4.31 & -0.24 & 11.2 & $0.99_{-0.05}^{+0.05}$ & $1.10_{-0.10}^{+0.13}$ & 1 & 1 & PC \\
\hline $\mathrm{C} 7$ & 215969174 & 14.3 & 5929 & 4.35 & 0.16 & 3.8 & $1.11_{-0.05}^{+0.05}$ & $1.15_{-0.09}^{+0.13}$ & 1 & 1 & PC \\
\hline $\mathrm{C} 7$ & 217393088 & 15.3 & 5839 & 4.09 & 0.25 & 5.1 & $1.19_{-0.08}^{+0.11}$ & $1.54_{-0.19}^{+0.22}$ & 1 & 1 & $\mathrm{PC}$ \\
\hline $\mathrm{C} 8$ & 220542353 & 8.8 & 6414 & 3.86 & -0.45 & 8.5 & $1.12_{-0.07}^{+0.09}$ & $1.91_{-0.25}^{+0.30}$ & 1 & 5 & $\mathrm{PC}$ \\
\hline $\mathrm{C} 8$ & 220383386 & 8.9 & 5305 & 4.47 & 0.11 & 0.0 & $0.89_{-0.03}^{+0.04}$ & $0.88_{-0.04}^{+0.06}$ & 1 & 1 & $\mathrm{PC}$ \\
\hline $\mathrm{C} 8$ & 220709978 & 9.4 & 5963 & 4.44 & -0.25 & 2.6 & $0.93_{-0.04}^{+0.04}$ & $0.96_{-0.06}^{+0.09}$ & 1 & 1 & $\mathrm{PC}$ \\
\hline $\mathrm{C} 8$ & 220303276 & 10.9 & 6446 & 4.29 & 0.15 & 10.7 & $1.31_{-0.05}^{+0.06}$ & $1.38_{-0.10}^{+0.14}$ & 1 & 3 & $\mathrm{PC}$ \\
\hline $\mathrm{C} 8$ & 220725183 & 11.5 & 6188 & 4.05 & -0.07 & 18.8 & $1.16_{-0.08}^{+0.10}$ & $1.58_{-0.19}^{+0.23}$ & 1 & 5 & PC \\
\hline $\mathrm{C} 8$ & 220376054 & 11.6 & 5863 & 4.19 & 0.06 & 2.5 & $1.05_{-0.06}^{+0.07}$ & $1.29_{-0.15}^{+0.18}$ & 1 & 1 & $\mathrm{PC}$ \\
\hline $\mathrm{C} 8$ & 220621788 & 11.8 & 5652 & 4.40 & 0.05 & 0.9 & $0.95_{-0.04}^{+0.05}$ & $0.99_{-0.07}^{+0.10}$ & 1 & 1 & $\mathrm{PC}$ \\
\hline $\mathrm{C} 8$ & 220674823 & 12.0 & 5547 & 4.44 & 0.14 & 1.4 & $0.96_{-0.04}^{+0.05}$ & $0.97_{-0.06}^{+0.09}$ & 1 & 1 & $\mathrm{PC}$ \\
\hline $\mathrm{C} 8$ & 220481411 & 12.1 & 4495 & $\ldots$ & 0.08 & $\ldots$ & .. & $0.71_{-0.10}^{+0.10}$ & 2 & 1 & $\mathrm{PC}$ \\
\hline $\mathrm{C} 8$ & 220294712 & 12.3 & 6100 & 4.36 & -0.11 & 4.3 & $1.04_{-0.05}^{+0.05}$ & $1.09_{-0.08}^{+0.12}$ & 1 & 1 & PC \\
\hline $\mathrm{C} 8$ & 220321605 & 12.6 & 4159 & $\ldots$ & -0.01 & $\ldots$ & $\ldots$ & $0.67_{-0.10}^{+0.10}$ & 2 & 1 & PC \\
\hline $\mathrm{C} 8$ & 220397060 & 12.8 & 5221 & 4.16 & -0.21 & 3.6 & $0.81_{-0.04}^{+0.11}$ & $0.84_{-0.07}^{+0.91}$ & 1 & 5 & $\mathrm{PC}$ \\
\hline $\mathrm{C} 8$ & 220187552 & 12.8 & 4197 & $\ldots$ & -0.02 & $\ldots$ & $\ldots$ & $0.66_{-0.10}^{+0.10}$ & 2 & 4 & $\mathrm{PC}$ \\
\hline $\mathrm{C} 8$ & 220621087 & 13.4 & 3633 & $\ldots$ & -0.28 & $\ldots$ & $\ldots$ & $0.45_{-0.10}^{+0.10}$ & 2 & 1 & $\mathrm{PC}$ \\
\hline $\mathrm{C} 8$ & 220504338 & 13.5 & 5648 & 4.18 & 0.30 & 2.0 & $1.08_{-0.06}^{+0.08}$ & $1.33_{-0.16}^{+0.18}$ & 1 & 1 & PC \\
\hline $\mathrm{C} 8$ & 220501947 & 13.5 & 4398 & $\ldots$ & 0.17 & $\ldots$ & $\ldots$ & $0.73_{-0.10}^{+0.10}$ & 2 & 1 & $\mathrm{PC}$ \\
\hline $\mathrm{C} 8$ & 220258394 & 13.7 & 5601 & 4.81 & 0.03 & 3.9 & $0.94_{-0.03}^{+0.04}$ & $0.87_{-0.04}^{+0.04}$ & 1 & 5 & $\mathrm{PC}$ \\
\hline $\mathrm{C} 8$ & 220554210 & 13.7 & 5440 & 4.47 & 0.16 & 1.2 & $0.94_{-0.04}^{+0.04}$ & $0.93_{-0.05}^{+0.07}$ & 1 & 1 & $\mathrm{PC}$ \\
\hline $\mathrm{C} 8$ & 220436208 & 13.9 & 5645 & 4.29 & 0.28 & 2.3 & $1.05_{-0.05}^{+0.06}$ & $1.17_{-0.12}^{+0.16}$ & 1 & 1 & PC \\
\hline $\mathrm{C} 8$ & 220629489 & 14.1 & 5050 & 4.47 & 0.32 & 2.7 & $0.88_{-0.03}^{+0.04}$ & $0.86_{-0.03}^{+0.05}$ & 1 & 1 & $\mathrm{PC}$ \\
\hline $\mathrm{C} 8$ & 220565349 & 14.1 & 5453 & 4.61 & -0.06 & 3.2 & $0.87_{-0.04}^{+0.004}$ & $0.84_{-0.03}^{+0.05}$ & 1 & 1 & $\mathrm{PC}$ \\
\hline $\mathrm{C} 8$ & 220209578 & 14.4 & 5757 & 4.47 & -0.26 & 3.8 & $0.87_{-0.04}^{+0.04}$ & $0.89_{-0.05}^{+0.07}$ & 1 & 1 & PC \\
\hline $\mathrm{C} 8$ & 220522262 & 14.8 & 4616 & $\ldots$ & 0.15 & $\ldots$ & $\ldots$ & $0.75_{-0.10}^{+0.10}$ & 2 & 1 & PC \\
\hline C6 & 212727070 & 9.4 & $\ldots$ & $\ldots$ & $\ldots$ & 20.1 & $\ldots$ & $\ldots$ & 0 & 3 & EB \\
\hline C6 & 212351868 & 10.2 & $\ldots$ & $\ldots$ & $\ldots$ & 46.0 & $\ldots$ & $\ldots$ & 0 & 3 & EB \\
\hline C6 & 212773309 & 11.4 & 5009 & 4.42 & 0.36 & 9.1 & $0.88_{-0.03}^{+0.03}$ & $0.86_{-0.04}^{+0.05}$ & 1 & 1 & EB \\
\hline $\mathrm{C} 7$ & 218541396 & 10.0 & $\ldots$ & $\ldots$ & $\ldots$ & 21.2 & $\ldots$ & $\ldots$ & 0 & 3 & EB \\
\hline C8 & 220666988 & 9.3 & $\ldots$ & $\ldots$ & $\ldots$ & 32.6 & $\ldots$ & $\ldots$ & 0 & 1 & EB \\
\hline $\mathrm{C} 8$ & 220431824 & 13.0 & 5434 & 4.10 & 0.04 & 8.6 & $0.95_{-0.03}^{+0.05}$ & $1.45_{-0.24}^{+0.19}$ & 1 & 1 & EB \\
\hline C5 & 212066407 & 12.2 & 5857 & 4.31 & -0.12 & 2.4 & $0.94_{-0.05}^{+0.05}$ & $1.07_{-0.10}^{+0.13}$ & 1 & 1 & EB \\
\hline $\mathrm{C} 6$ & 212651120 & 10.5 & $\ldots$ & $\ldots$ & $\ldots$ & 128.0 & . & $\ldots$ & 0 & 3 & EB \\
\hline $\mathrm{C} 5$ & 211993818 & 7.2 & 5228 & 3.06 & -0.08 & 6.1 & $2.09_{-0.26}^{+0.21}$ & $8.03_{-2.32}^{+1.18}$ & 1 & 5 & Other \\
\hline
\end{tabular}


Table 2

(Continued)

\begin{tabular}{|c|c|c|c|c|c|c|c|c|c|c|c|}
\hline Camp. & EPIC & $\begin{array}{c}K p \\
(\mathrm{mag})\end{array}$ & $\begin{array}{l}T_{\text {eff }} \\
(\mathrm{K})\end{array}$ & $\begin{array}{l}\log g \\
(\operatorname{cgs})\end{array}$ & $\begin{array}{c}{[\mathrm{Fe} / \mathrm{H}]} \\
(\mathrm{dex})\end{array}$ & $\begin{array}{c}v \sin i \\
\left(\mathrm{~km} \mathrm{~s}^{-1}\right)\end{array}$ & $\begin{array}{l}M_{\star} \\
M_{\odot}\end{array}$ & $\begin{array}{l}R_{\star} \\
R_{\odot}\end{array}$ & $\mathrm{SM}^{\mathrm{a}}$ & $\mathrm{SB} 2^{\mathrm{b}}$ & $\operatorname{disp}^{c}$ \\
\hline $\mathrm{C} 5$ & 211311380 & 9.1 & 6251 & 4.28 & 0.00 & 5.7 & $1.15_{-0.05}^{+0.06}$ & $1.25_{-0.11}^{+0.16}$ & 1 & 1 & Other \\
\hline $\mathrm{C} 5$ & 211886472 & 11.1 & 6458 & 4.32 & -0.04 & 8.0 & $1.20_{-0.05}^{+0.05}$ & $1.26_{-0.08}^{+0.13}$ & 1 & 1 & Other \\
\hline $\mathrm{C} 5$ & 211987231 & 11.7 & 6103 & 4.44 & -0.04 & 5.5 & $1.07_{-0.05}^{+0.05}$ & $1.08_{-0.07}^{+0.09}$ & 1 & 4 & Other \\
\hline $\mathrm{C} 5$ & 211941472 & 11.8 & 5767 & 4.06 & -0.00 & 2.6 & $1.01_{-0.06}^{+0.07}$ & $1.45_{-0.18}^{+0.22}$ & 1 & 1 & Other \\
\hline $\mathrm{C} 5$ & 211770696 & 12.3 & 5786 & 4.22 & -0.31 & 1.5 & $0.86_{-0.03}^{+0.04}$ & $1.09_{-0.13}^{+0.18}$ & 1 & 1 & Other \\
\hline $\mathrm{C} 5$ & 212006318 & 12.9 & 5822 & 4.11 & 0.03 & 2.1 & $1.03_{-0.06}^{+0.07}$ & $1.39_{-0.17}^{+0.20}$ & 1 & 1 & Other \\
\hline $\mathrm{C} 5$ & 211978909 & 13.2 & 5112 & 3.70 & -0.33 & 1.9 & $0.96_{-0.07}^{+0.14}$ & $2.41_{-0.28}^{+0.39}$ & 1 & 1 & Other \\
\hline $\mathrm{C} 5$ & 211825866 & 13.8 & 5279 & 4.74 & -0.19 & 0.9 & $0.80_{-0.03}^{+0.03}$ & $0.76_{-0.02}^{+0.03}$ & 1 & 1 & Other \\
\hline C6 & 212473154 & 9.0 & 4740 & 2.68 & 0.01 & 4.3 & $1.85_{-0.37}^{+0.50}$ & $10.92_{-1.74}^{+0.90}$ & 1 & 1 & Other \\
\hline C6 & 212768333 & 11.0 & 5242 & 4.70 & 0.05 & 5.8 & $0.86_{-0.03}^{+0.03}$ & $0.81_{-0.03}^{+0.04}$ & 1 & 1 & Other \\
\hline C6 & 212705192 & 11.7 & $\ldots$ & $\ldots$ & $\ldots$ & 20.8 & $\ldots$ & $\ldots$ & 0 & 5 & Other \\
\hline C7 & 213743957 & 11.4 & $\ldots$ & $\ldots$ & $\ldots$ & 62.5 & $\ldots$ & $\ldots$ & 0 & 3 & Other \\
\hline $\mathrm{C} 7$ & 215346008 & 11.8 & 4378 & 2.36 & -0.02 & 5.1 & $1.23_{-0.23}^{+0.47}$ & $12.34_{-1.61}^{+2.96}$ & 1 & 1 & Other \\
\hline C7 & 216050437 & 12.3 & $\ldots$ & $\ldots$ & $\ldots$ & 38.5 & $\ldots$ & $\ldots$ & 0 & 5 & Other \\
\hline $\mathrm{C} 7$ & 218212249 & 13.1 & 4379 & 2.25 & -0.54 & 5.1 & $1.01_{-0.13}^{+0.33}$ & $13.91_{-1.91}^{+2.79}$ & 1 & 1 & Other \\
\hline $\mathrm{C} 8$ & 220503133 & 6.5 & 4776 & 2.68 & 0.03 & 4.5 & $1.97_{-0.32}^{+0.58}$ & $10.99_{-1.47}^{+1.25}$ & 1 & 1 & Other \\
\hline $\mathrm{C} 8$ & 220492184 & 8.0 & 6694 & 3.34 & -0.81 & 15.3 & $1.64_{-0.12}^{+0.15}$ & $4.12_{-0.57}^{+0.73}$ & 1 & 5 & Other \\
\hline $\mathrm{C} 8$ & 220493203 & 9.9 & 5054 & 2.92 & -0.46 & 3.9 & $1.70_{-0.56}^{+0.39}$ & $7.88_{-2.21}^{+1.21}$ & 1 & 1 & Other \\
\hline $\mathrm{C} 8$ & 220643470 & 10.8 & 4432 & 2.34 & -0.69 & 4.2 & $0.96_{-0.11}^{+0.24}$ & $12.19_{-1.39}^{+2.16}$ & 1 & 1 & Other \\
\hline $\mathrm{C} 8$ & 220487418 & 12.1 & 6029 & 4.24 & 0.05 & 4.5 & $1.10_{-0.06}^{+0.07}$ & $1.27_{-0.14}^{+0.19}$ & 1 & 1 & Other \\
\hline $\mathrm{C} 8$ & 220209709 & 12.2 & 5911 & 4.14 & 0.02 & 1.6 & $1.06_{-0.06}^{+0.08}$ & $1.37_{-0.17}^{+0.19}$ & 1 & 1 & Other \\
\hline $\mathrm{C} 8$ & 220650439 & 12.2 & 5676 & 4.38 & 0.13 & 1.3 & $0.99_{-0.05}^{+0.05}$ & $1.04_{-0.08}^{+0.11}$ & 1 & 1 & Other \\
\hline
\end{tabular}

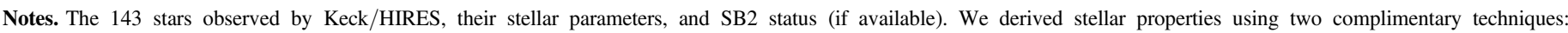

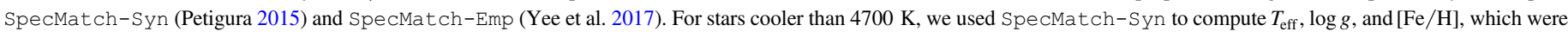

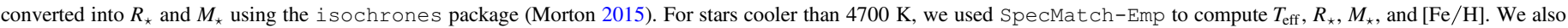
searched for SB2s using the methodology of Kolbl et al. (2015).

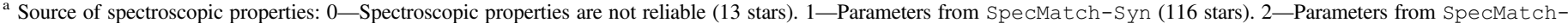

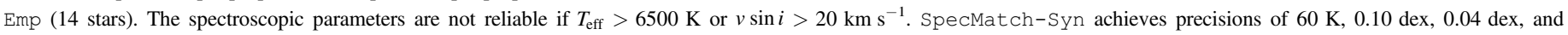
$1 \mathrm{~km} \mathrm{~s}^{-1}$ in $T_{\text {eff }}, \log g,[\mathrm{Fe} / \mathrm{H}]$, and $v \sin i$. SpecMatch-Emp achieves precisions of $70 \mathrm{~K}$ in $T_{\text {eff }}, 10 \%$ in $R_{\star}$, and $0.12 \mathrm{dex}$ in [Fe/H].

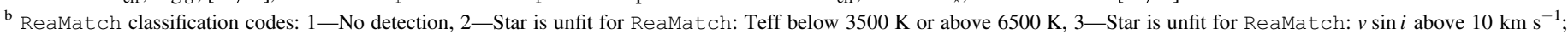
4-Ambiguous detection; 5-Obvious detection.

${ }^{\mathrm{c}}$ Flag indicating whether the target is a planet candidate (PC), eclipsing binary (EB), or is not included in our candidate list (Other).

(This table is available in machine-readable form.)

Gaussian constraints on $T_{\text {eff }}, \log g$, and $[\mathrm{Fe} / \mathrm{H}]$. For consistency, we used $T_{\text {eff }}, \log g$, and $[\mathrm{Fe} / \mathrm{H}]$ constrained by broadband photometry from Huber et al. (2016). While photometrically constrained $\log g$ and $[\mathrm{Fe} / \mathrm{H}]$ are low-precision, $u$ is only weakly dependent on these parameters, and the derived transit parameters are only weakly dependent on $u$.

8. Dilution. Log-uniform prior, $\log \delta=\left[10^{-6}, 10^{0}\right]$. Our fits do not incorporate dilution constraints, so $\delta$ always reverts to the prior. We include $\delta$ so we can incorporate dilution constraints at later times. ${ }^{20}$

\footnotetext{
${ }^{20}$ The non-zero prior on $\delta$ slightly alters the derived value of $R_{P} / R_{\star}$. However, because the median $\delta$ is $10^{-3}$, this amounts to a fractional change in derived radius ratio of $\Delta\left(R_{P} / R_{\star}\right) /\left(R_{P} / R_{\star}\right) \approx \delta / 2=5 \times 10^{-4}$, which may be ignored.
}

In Table 1 , we report $1 \sigma$ credible ranges on $P, T_{0}, R_{P} / R_{\star}$, transit duration $T_{14}$, and impact parameter $b$.

\section{Spectroscopy}

\subsection{Spectroscopic Follow Program}

As a part of our team's standard follow-up efforts, we obtained optical spectra of 143 C5-C8 target stars using the HIgh Resolution Echelle Spectrometer (HIRES; Vogt et al. 1994) on the Keck I 10 m telescope. We gathered spectra for the purpose of improving host star parameters and to place limits on the presence of companion stars with small separations through searches for spectroscopic binaries (SB2s). We aimed to obtain a spectrum of every K2OI brighter than $V=14.0 \mathrm{mag}$. For $\mathrm{G}$ stars, this limit corresponds roughly to $K p=13.6 \mathrm{mag}$. 


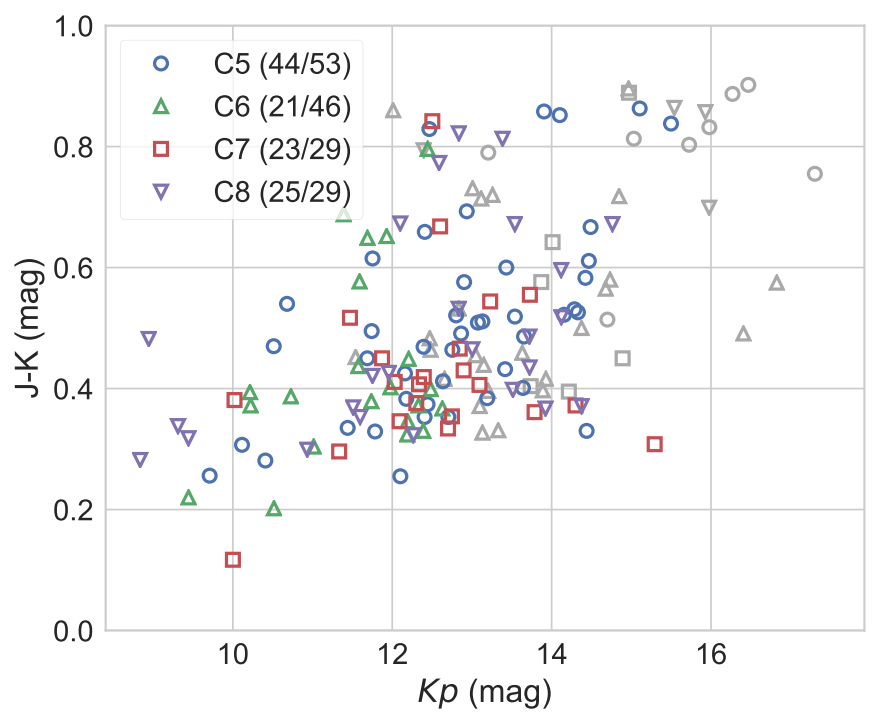

Figure 2. Distribution of K2OIs as a function of $K p$ and $J-K$ color. The colored/gray points represent targets with/without a HIRES spectrum. The marker color and shape represent a target's specific $K 2$ campaign. The HIRES follow up is nearly complete to $K p=14$ mag.

Table 2 lists the C5-C8 targets that we observed with HIRES, along with the results from our stellar characterization and search for spectroscopic binaries, which are described in Sections 3.2 and 3.3, respectively. We obtained HIRES spectra of 105/141 of the planet candidate host stars and for 8/16 of the likely EBs. In addition, we observed 30 other $\mathrm{C} 5-\mathrm{C} 8$ targets that we did not identify as candidates. These were typically observed because they were identified as planet candidates by other groups.

We used the HIRES exposure meter to obtain consistent signal-to-noise ratio $(\mathrm{S} / \mathrm{N})$ levels depending on $V$-band apparent magnitude: $\mathrm{S} / \mathrm{N}=45(V<13.0 \mathrm{mag}), \mathrm{S} / \mathrm{N}=32$ $(V=13.0-14.0 \mathrm{mag})$, and $\mathrm{S} / \mathrm{N}=20(V>14.0 \mathrm{mag})$. Exposure times ranged from $\approx 10 \mathrm{~s}$ for $V=9$ mag targets to $\approx 400 \mathrm{~s}$ for $V=15$ mag targets. $\mathrm{S} / \mathrm{N}$ is computed per reduced HIRES pixel on blaze at $5500 \AA$. Our HIRES follow up was nearly complete down to $V=14 \mathrm{mag}$. Figure 2 shows the distribution of candidate hosts as a function of $K p$ and $J-K$ color. The candidates with HIRES spectra are labeled. Figure 3 shows a spectral segment for one K2OI to illustrate typical spectral resolution and $\mathrm{S} / \mathrm{N}$ level.

\subsection{Stellar Characterization}

We used our spectra to improve the precision of stellar and planetary properties such as $R_{\star}$ and $R_{P}$. We analyzed each spectrum with one of two related publicly available codes: SpecMatch-Syn (Petigura 2015) ${ }^{21}$ and SpecMatch-Emp (Yee et al. 2017). ${ }^{22}$

SpecMatch-Syn fits five regions of optical spectrum by interpolating within a grid of model spectra from Coelho et al. (2005). Recently, SpecMatch-Syn enabled a homogeneous analysis of 1305 spectra of planet hosts identified during the prime Kepler mission (Petigura et al. 2017). For stars with $T_{\mathrm{eff}}=4700-6500 \mathrm{~K}$ and $v \sin i<20 \mathrm{~km} \mathrm{~s}^{-1}$, SpecMatchSyn achieves precisions of $60 \mathrm{~K}$ in $T_{\text {eff }}, 0.10 \operatorname{dex}$ in $\log g$, and $0.04 \mathrm{dex}$ in $[\mathrm{Fe} / \mathrm{H}]$, and $1 \mathrm{~km} \mathrm{~s}^{-1}$ in $v \sin i$.

\footnotetext{
21 https://github.com/petigura/specmatch-syn

22 https://github.com/samuelyeewl/specmatch-emp
}

We converted $T_{\text {eff }}, \log g$, and $[\mathrm{Fe} / \mathrm{H}]$ into $M_{\star}$ and $R_{\star}$ using the publicly available isoclassify Python package (Huber et al. 2017), ${ }^{23}$ which uses the MESA Isochrones and Stellar Tracks (MIST) database (Paxton et al. 2011, 2013, 2015; Choi et al. 2016). While SpecMatch-Syn returns $T_{\text {eff }}$ precise to $60 \mathrm{~K}$, as tested against other spectral synthesis codes, there are known offsets between spectroscopic $T_{\text {eff }}$ and other techniques such as the Infrared Flux Method (IRFM) and interferometry. For a detailed discussion of different $T_{\text {eff }}$ scales, see Brewer et al. (2016). To account for systematic uncertainties associated with the spectroscopic $T_{\text {eff }}$ scale, we have increased the $T_{\text {eff }}$ uncertainties to $100 \mathrm{~K}$ during the isochrone modeling.

The radius uncertainties derived from specMatch-Syn parameters do not incorporate uncertainties associated with the MIST models themselves. Johnson et al. (2017) estimated the size of these model-dependent uncertainties through a comparison of stellar radii derived using MIST models and Dartmouth Stellar Evolution Program models (Dotter et al. 2008) with identical inputs. They estimated that model-dependent radius errors are $\approx 2 \%$ for dwarf stars $(\log g<3.9)$ and $\approx 10 \%$ for evolved stars $(\log g>3.9)$. These model-dependent uncertainties are typically smaller than the formal radius uncertainties returned by isoclassify.

For $T_{\text {eff }} \lesssim 4700 \mathrm{~K}$, SpecMatch-Syn does not return reliable parameters, due to the onset of molecular lines that are not welltreated in the Coelho et al. (2005) models. While the highresolution optical spectra of stars later than $\sim \mathrm{K} 4$ are challenging to compute directly, their spectra contain a wealth of information, which can be used to constrain stellar properties. SpecMatchEmp circumvents the challenges in spectral synthesis by matching a target spectrum against an empirical spectra library of $\approx 400$ touchstone stars with well-known parameters measured through other methods such as SED-fitting, interferometry, or the IRFM. SpecMatch-Emp interpolates between this library of empirical spectra to find a linear combination of library spectra that best reproduces the target spectrum. Figure 3 shows the best-fitting linear combination of library spectrum for EPIC 212006344, an M0 dwarf. The derived stellar parameters for the target spectrum are the weighted average of the $T_{\text {eff }}, R_{\text {star }}$, and fe of the five bestfitting library spectra Figure 4. SpecMatch-Emp achieves precisions of $70 \mathrm{~K}$ in $T_{\text {eff }}, 10 \%$ in $R_{\star}$, and $0.12 \mathrm{dex}$ in $[\mathrm{Fe} / \mathrm{H}]$.

We adopt parameters from SpecMatch-Syn for stars hotter than $4700 \mathrm{~K}^{24}$ and SpecMatch-Emp for cooler stars. Figure 5 shows the $T_{\text {eff }}$ and $R_{\star}$ for K2OIs with reliable spectroscopic parameters. Our adopted stellar parameters are listed in Table 2. Our team also conducts a parallel characterization of cool stars using NIR spectroscopy. Stellar properties up through campaign 7 are given in Martinez et al. (2017) and Dressing et al. (2017).

\subsection{Searches for Spectroscopic Binaries}

Each HIRES spectrum is methodically searched for secondary spectral lines using the ReaMatch algorithm (Kolbl et al. 2015). To identify secondary spectra, each spectrum is first cross-correlated against a set of previously observed spectra. This catalog has spectra with $T_{\text {eff }}=3500-$ $6500 \mathrm{~K}$ and $\log g=3.0-4.5 \mathrm{dex}$. The best-matching spectrum is subtracted from the target spectrum and the residuals are again cross-correlated with the catalog spectra.

\footnotetext{
23 https://github.com/danxhuber/isoclassify

24 As measured by SpecMatch-Emp.
} 


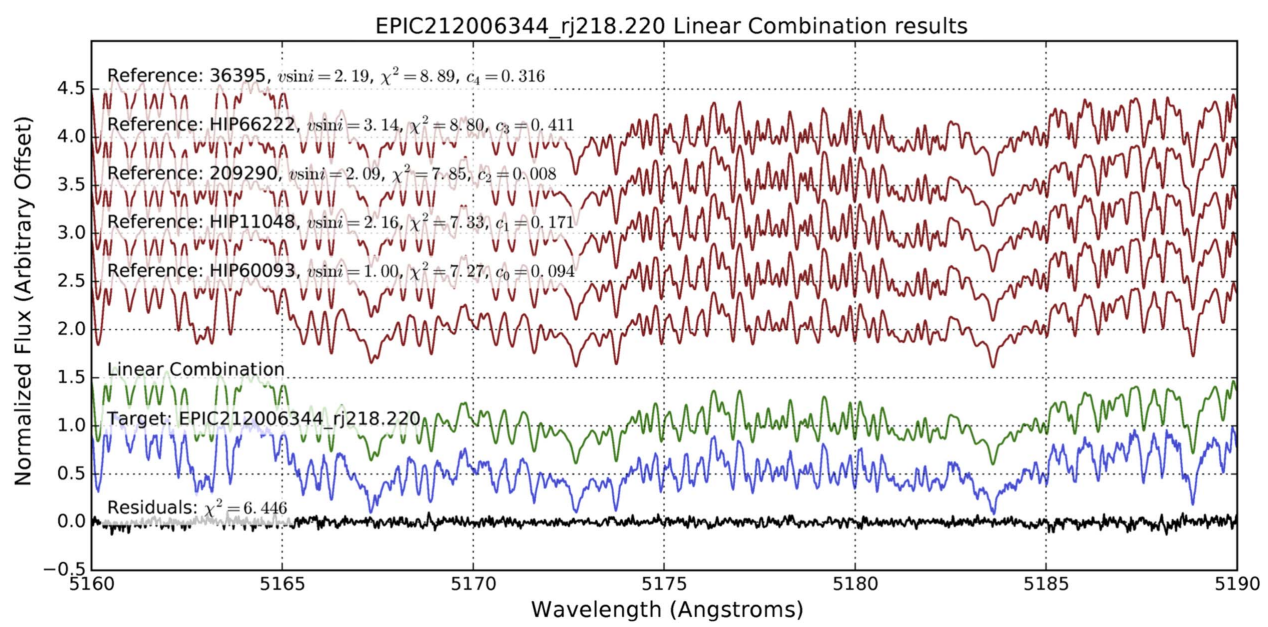

Figure 3. Example HIRES characterization spectrum with SpecMatch-Emp fit. Blue spectrum is of EPIC 212006344, an M0 dwarf, and illustrates the typical S/N from our characterization program of $\approx 45 /$ pixel. The spectrum contains a dense forest of molecular lines, making ab initio spectral synthesis challenging. Red spectra are drawn from SpecMatch-Emp library and are identified as similar to the target spectrum by the SpecMatch-Emp algorithm. The green and black spectra are the best-fitting linear combination spectrum and residual spectrum, respectively.

EPIC212006344_rj218.220 references used in linear combination
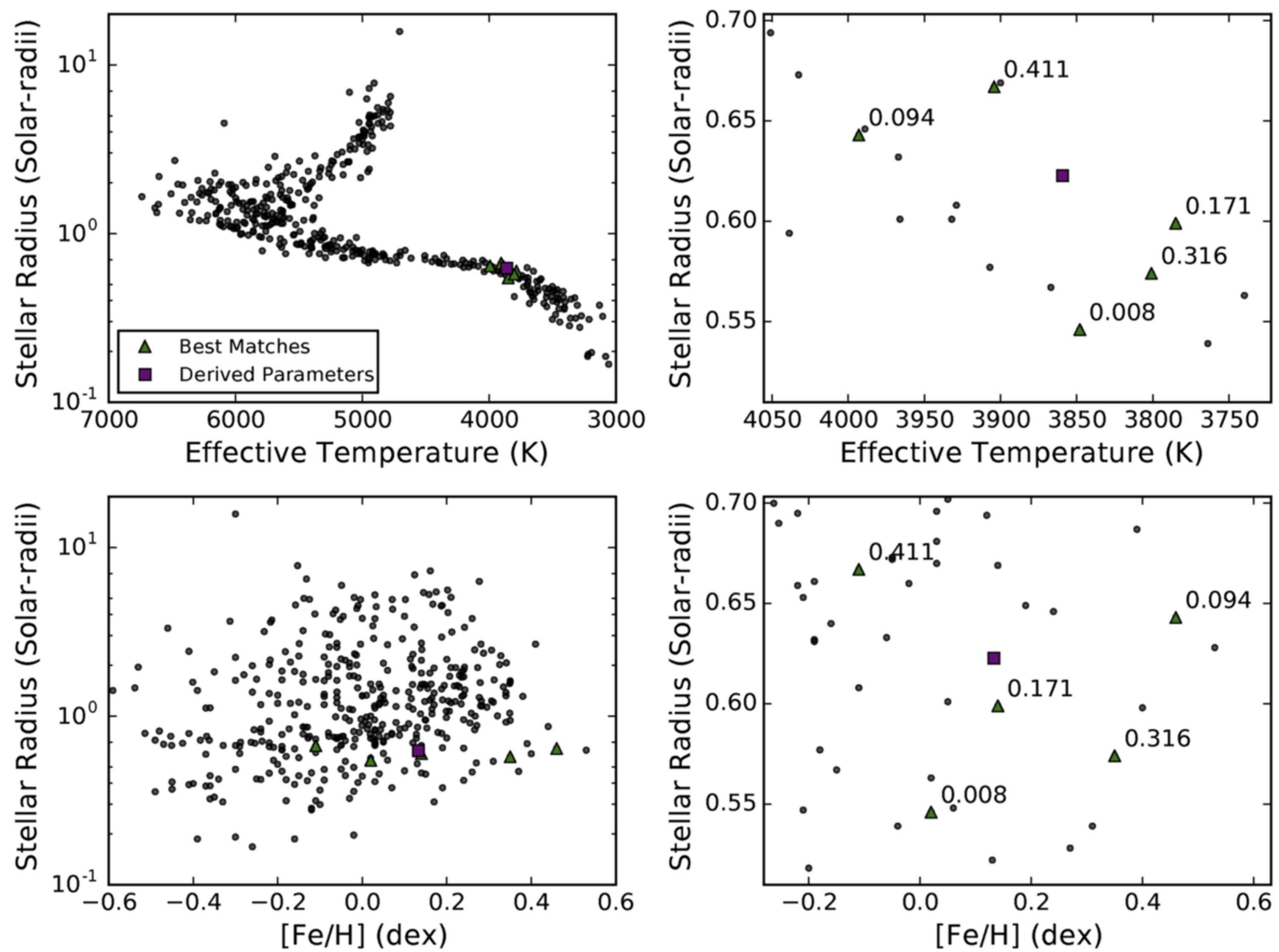

Figure 4. SpecMatch-Emp characterization of EPIC 212006344. Black points: $T_{\text {eff }}, R_{\star}$, and [Fe/H] from the SpecMatch-Emp library. Green triangles: properties of the closest-matching library spectra (red spectra in Figure 3). Purple square: linear combination of library spectra spectra. The final derived parameters are $T_{\text {eff }}=3925 \pm 70 \mathrm{~K},[\mathrm{Fe} / \mathrm{H}]=0.43 \pm 0.12 \mathrm{dex}, R_{\star}=0.63 \pm 0.10 \mathrm{dex}$.

ReaMatch is sensitive to companions down to $1 \%$ the brightness of the primary having RV offsets $|\Delta v|>$ $10 \mathrm{~km} \mathrm{~s}^{-1}$. Optimized for slowly rotating FGKM stars, ReaMatch is insensitive to SB2s orbiting primaries with $v \sin i>10 \mathrm{~km} \mathrm{~s}^{-1}$ or with $T_{\text {eff }}$ outside $3500-6500 \mathrm{~K}$. Table 2 lists the results of our SB2 search.

\section{Planet Candidates}

We list the 151 planet candidates and 16 likely EBs in Table 1 . We compute planetary radius by combining $R_{P} / R_{\star}$ measured from the light curve with the best available $R_{\star}$. For the stars without spectra, we estimate $R_{\star}$ from broadband 


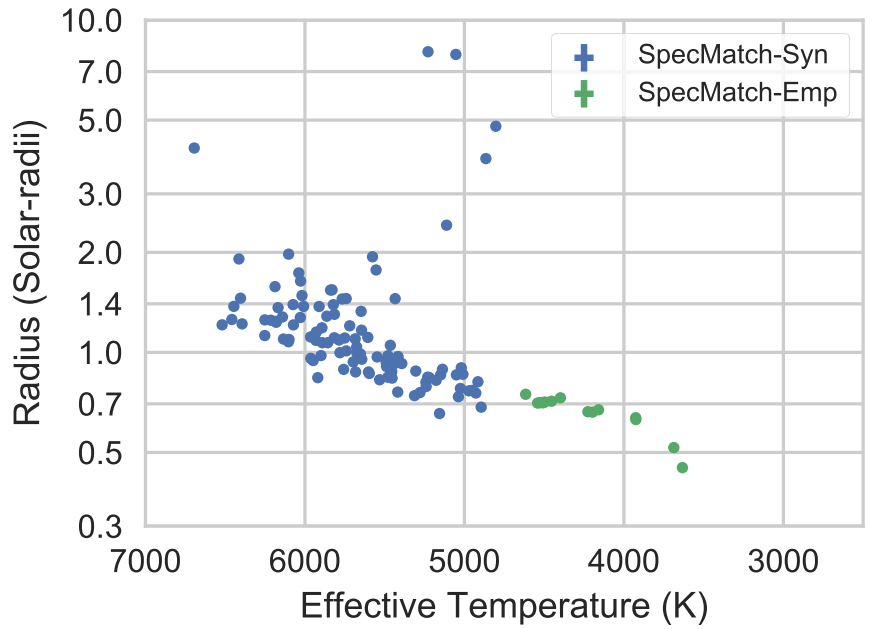

Figure 5. K2OIs with reliable effective temperatures and stellar radii, as measured by SpecMatch-Syn (blue) or SpecMatch-Emp (green). Median uncertainties are shown at the top right.

photometry, according to the following procedure: we estimate spectral types (SpTs) using tabulated photometric relations (Kraus \& Hillenbrand 2007; Pecaut \& Mamajek 2013; Rodriguez et al. 2013) and convert SpTs into $R_{\star}$ based on interferometric studies (Boyajian et al. 2012). These stellar radii are crude and we estimate their typical uncertainties to be $\approx 40 \%$, typical of errors derived from broadband photometry (Brown et al. 2011).

Figure 6 shows the distribution of the 151 planet candidates in the $P-R_{P}$ plane. Figure 7 shows one-dimensional histograms of our candidates as a function of $P, R_{P}$, and $K p$. The median host star is nearly two magnitudes brighter in the Kepler bandpass than the median KOI from the prime Kepler mission (12.8 mag versus $14.6 \mathrm{mag}$, Mullally et al. 2015). Our candidates have the following multiplicity distribution: 132 singles, 8 doubles, and 1 triple planet system.

We consulted the NASA Exoplanet Archive (NEA; Akeson et al. 2013) ${ }^{25}$ to check whether previous analyses have reported significant numbers of candidates presented in this work. Of the catalogs incorporated into the NEA as of 2017-11-09, Barros et al. (2016; B16 hereafter) and Pope et al. (2016; P16 hereafter) included 10 or more candidates from C5-C8.

B16 reported 172 planet candidates from $\mathrm{C} 1-\mathrm{C} 6$, of which 86 were in C5 and C6. Our catalog contains 107 candidates from $\mathrm{C} 5$ and C6. The two catalogs share 49 candidates. There are 58 candidates in our catalog that are not in B16, and there are 37 candidates in B16 that are not in our catalog.

P16 reported 168 planet candidates in C5 and C6. Of these, our catalog includes 73 candidates. There are 34 candidates in our catalog that are not in P16, and there are 95 candidates in P16 that are not in our catalog.

As a final point of comparison, B16 and P16 share 59 candidates. There are 27 candidates in B16 that are not in P16, and there are 109 candidates in P16 that were not in B16. Figure 8 is a Venn diagram that summarizes the degree of overlap between the various samples.

A detailed analysis of why any particular candidate appeared in one catalog and not another is beyond the scope of this work. Broadly speaking, the lack of perfect overlap likely arises due to differences in photometric extraction algorithms,

\footnotetext{
${ }^{25}$ https://exoplanetarchive.ipac.caltech.edu/
}

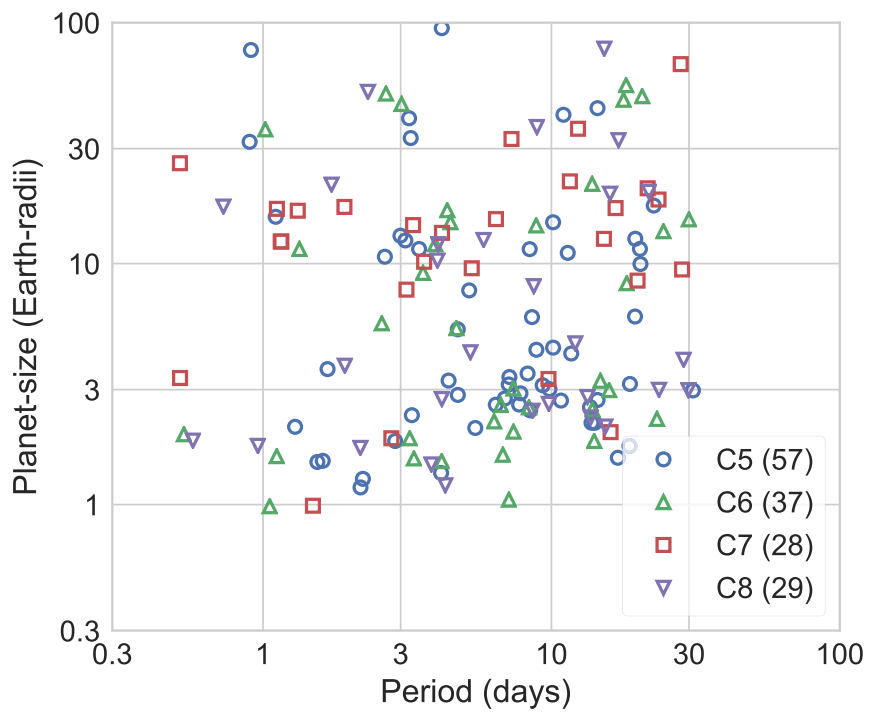

Figure 6. Planet size and orbital period for 151 planet candidates identified by $K 2$ in $\mathrm{C} 5-\mathrm{C} 8$. We have excluded targets identified as likely EBs from their light curve morphology. The legend at lower right links marker shape/color to a specific campaign and gives the total number of candidates identified in each campaign.

transit search algorithms, adopted signal-to-noise threshold for candidate status, and vetting diagnostics.

\section{Summary}

We report 151 planet candidates orbiting 141 stars detected through a systematic search of $K 2$ photometry from campaigns C5-C8. We also identified 16 likely EBs based on their light curve morphology. We obtained Keck/HIRES optical spectra of 105/141 planet candidate host stars and 8/16 EBs to improve our understanding of host star and planet properties and to search for binary companions.

A small fraction of our planet candidates reside in multicandidate systems ( 8 doubles and 1 triple). These systems have a low false-positive probability $(\lesssim 1 \%)$ due to their multiplicity (Lissauer et al. 2012). The remaining 132 candidates are wellvetted and well-characterized planet candidates, but have yet been confirmed or statistically validated. Statistical validation requires a detailed analysis of light curve shape and constraints on the presence of blends from high-resolution imaging. Crossfield et al. (2016) performed such an analysis to validate 104 K2 planet candidates identified during C0-C4. Our team's high-contrast imaging follow up will be presented in E. Gonzales et al. (2017, in preparation). An analysis of the false-positive probabilities of our candidates will be presented in J. Livingston et al. (2017, in preparation).

Our typical candidate is two magnitudes brighter than the typical candidate from the Kepler prime mission due to the larger region of sky observed by $K 2$. As a result, these candidates make up a valuable sample for further characterization efforts, such as Doppler measurements of planet masses.

We thank the anonymous referee for a thoughtful reading of the manuscript and for useful suggestions. E.A.P. acknowledges support from Hubble Fellowship grant HST-HF251365.001-A awarded by the Space Telescope Science Institute, which is operated by the Association of Universities for Research in Astronomy, Inc. for NASA under contract NAS 

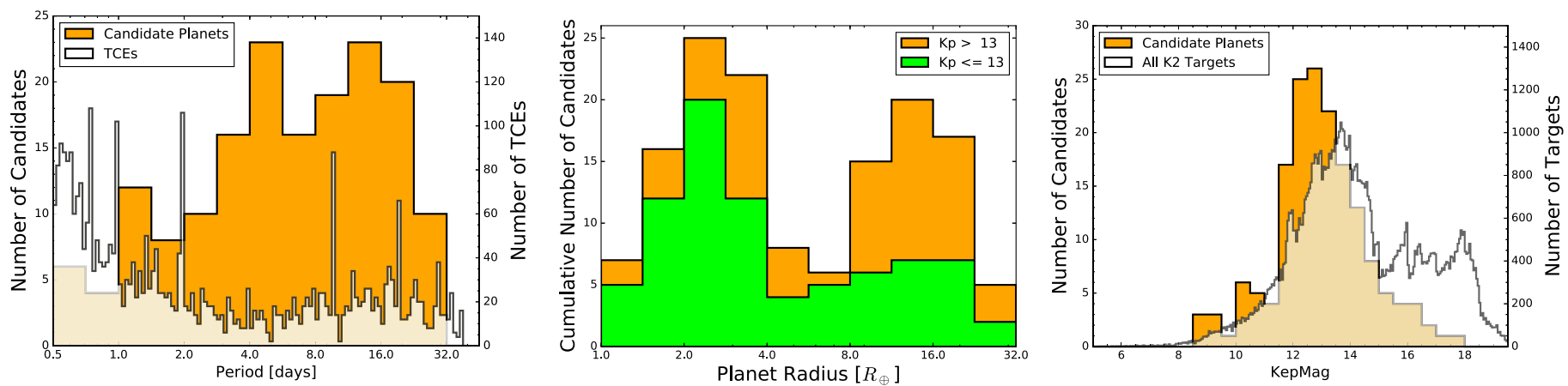

Figure 7. Left: orbital periods of transit-like signals identified in our analysis. The orange histogram (axis at left) indicates the distribution of planet candidates. The pale, narrow-binned histogram (axis at right) indicates the Threshold-crossing Events (TCEs) identified by TERRA in our initial transit search with MES $\geqslant 10$. Middle: cumulative histograms of radii for our planet candidates. Most are moderately bright at $K p \leqslant 13 \mathrm{mag}$, but at large radii over half orbit fainter stars; a large fraction of this second group are likely false positives. Right: the orange histogram (axis at left) shows the distribution of $K p$ for planet candidates. For comparison, the pale histogram (axis at right) shows all target stars from C5-C8.

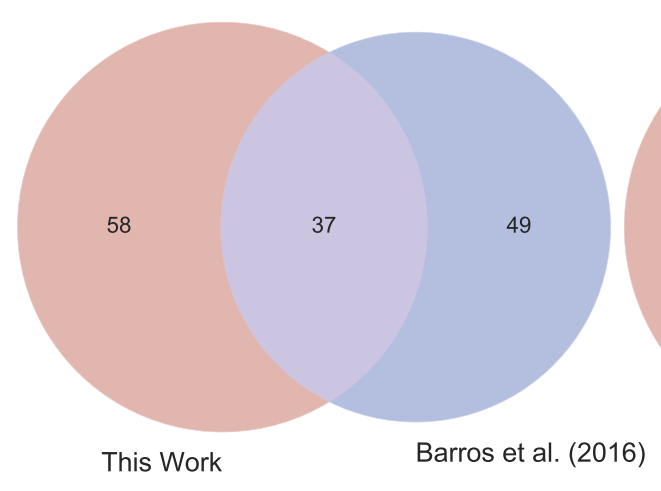

This Work
Barros et al. (2016)

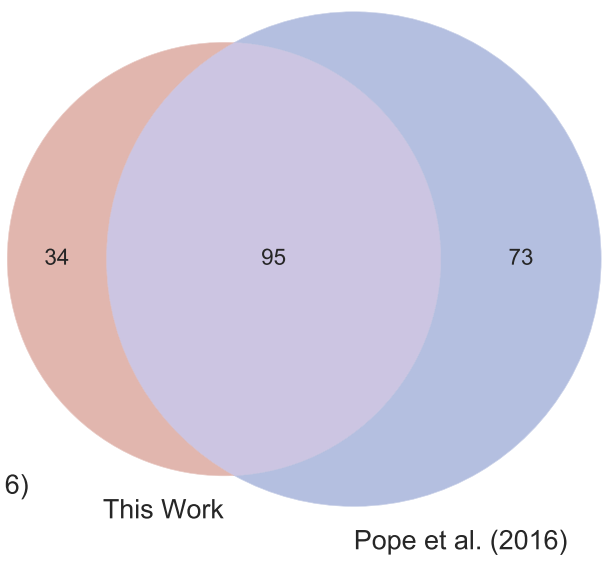

Pope et al. (2016)

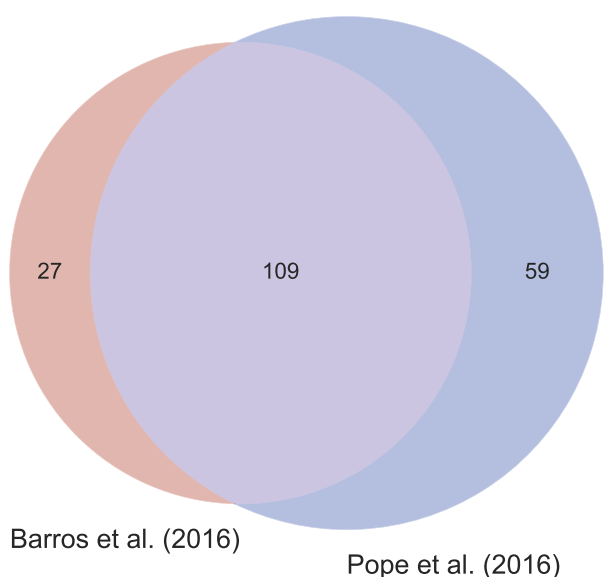

Pope et al. (2016)

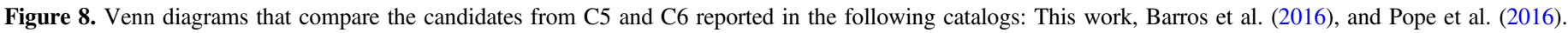

5-26555. Work by C.D.D. was performed in part under contract with the Jet Propulsion Laboratory (JPL) funded by NASA through the Sagan Fellowship Program executed by the NASA Exoplanet Science Institute. This research used the computing resources of NERSC, a DOE Office of Science User Facility supported by the Office of Science of the U.S. Department of Energy under Contract No. DE-AC02-05CH11231. Finally, the authors wish to recognize and acknowledge the very significant cultural role and reverence that the summit of Maunakea has always had within the indigenous Hawaiian community. We are most fortunate to have had the opportunity to conduct observations from this mountain.

Software: batman (Kreidberg 2015), SpecMatch-Syn (Petigura 2015), SpecMatch-Emp (Yee et al. 2017), k2phot (https://github.com/petigura/k2phot), isoclassify (Huber et al. 2017), isochrones (Morton 2015).

\section{ORCID iDs}

Erik A. Petigura (i) https://orcid.org/0000-0003-0967-2893

Howard Isaacson (1) https://orcid.org/0000-0002-0531-1073

Jessie L. Christiansen (1) https://orcid.org/0000-0002-

8035-4778

Courtney D. Dressing (i) https://orcid.org/0000-00018189-0233

Benjamin J. Fulton (10) https://orcid.org/0000-0003-3504-5316

Andrew W. Howard 주 https://orcid.org/0000-0001-

8638-0320
Molly R. Kosiarek i https://orcid.org/0000-0002-6115-4359

Evan Sinukoff (i) https://orcid.org/0000-0002-5658-0601

Samuel W. Yee iㅏ https://orcid.org/0000-0001-7961-3907

\section{References}

Aigrain, S., Hodgkin, S. T., Irwin, M. J., Lewis, J. R., \& Roberts, S. J. 2015 MNRAS, 447, 2880

Akeson, R. L., Chen, X., Ciardi, D., et al. 2013, PASP, 125, 989

Barros, S. C. C., Demangeon, O., \& Deleuil, M. 2016, A\&A, 594, A100

Borucki, W. J., Koch, D., Basri, G., et al. 2010, Sci, 327, 977

Boyajian, T. S., von Braun, K., van Belle, G., et al. 2012, ApJ, 757, 112

Brewer, J. M., Fischer, D. A., Valenti, J. A., \& Piskunov, N. 2016, ApJS, 225,32

Brown, T. M., Latham, D. W., Everett, M. E., \& Esquerdo, G. A. 2011, AJ, 142,112

Choi, J., Dotter, A., Conroy, C., et al. 2016, ApJ, 823, 102

Christiansen, J. L., Crossfield, I. J. M., Barentse, G., et al. 2017, AJ, in press Christiansen, J. L., Jenkins, J. M., Caldwell, D. A., et al. 2012, PASP, 124, 1279

Coelho, P., Barbuy, B., Meléndez, J., Schiavon, R. P., \& Castilho, B. V. 2005 , A\&A, 443, 735

Crossfield, I. J. M., Ciardi, D. R., Petigura, E. A., et al. 2016, ApJS, 226, 7 Crossfield, I. J. M., Petigura, E., Schlieder, J. E., et al. 2015, ApJ, 804, 10

David, T. J., Hillenbrand, L. A., Petigura, E. A., et al. 2016, Natur, 534, 658 Deming, D., Knutson, H., Kammer, J., et al. 2015, ApJ, 805, 132

Dotter, A., Chaboyer, B., Jevremović, D., et al. 2008, ApJS, 178, 89

Dressing, C. D., \& Charbonneau, D. 2015, ApJ, 807, 45

Dressing, C. D., Newton, E. R., Schlieder, J. E., et al. 2017, ApJ, 836, 167

Eastman, J., Gaudi, B. S., \& Agol, E. 2013, PASP, 125, 83

Foreman-Mackey, D., Montet, B. T., Hogg, D. W., et al. 2015, ApJ, 806, 215 
Goodman, J., \& Weare, J. 2010, Communications in Applied Mathematics and Computational Science, 5, 65

Howard, A. W., Sanchis-Ojeda, R., Marcy, G. W., et al. 2013, Natur, 503, 381

Howell, S. B., Sobeck, C., Haas, M., et al. 2014, PASP, 126, 398

Huber, D., Bryson, S. T., Haas, M. R., et al. 2016, ApJS, 224, 2

Huber, D., Zinn, J., Bojsen-Hansen, M., et al. 2017, ApJ, 844, 102

Johnson, J. A., Petigura, E. A., Fulton, B. J., et al. 2017, AJ, 154, 108

Jontof-Hutter, D., Rowe, J. F., Lissauer, J. J., Fabrycky, D. C., \& Ford, E. B. 2015, Natur, 522, 321

Kolbl, R., Marcy, G. W., Isaacson, H., \& Howard, A. W. 2015, AJ, 149, 18

Kraus, A. L., \& Hillenbrand, L. A. 2007, AJ, 134, 2340

Kreidberg, L. 2015, PASP, 127, 1161

Lissauer, J. J., Marcy, G. W., Rowe, J. F., et al. 2012, ApJ, 750, 112

Lucy, L. B., \& Sweeney, M. A. 1971, AJ, 76, 544

Luger, R., Agol, E., Kruse, E., et al. 2016, AJ, 152, 100

Mann, A. W., Newton, E. R., Rizzuto, A. C., et al. 2016, AJ, 152, 61

Marcy, G. W., Isaacson, H., Howard, A. W., et al. 2014, ApJS, 210, 20

Martinez, A. O., Crossfield, I. J. M., Schlieder, J. E., et al. 2017, ApJ, 837, 72

Montet, B. T., Morton, T. D., Foreman-Mackey, D., et al. 2015, ApJ, 809, 25

Morton, T. D. 2015, Isochrones: Stellar Model Grid Package, Astrophysics Source Code Library, ascl:1503.010

Mullally, F., Coughlin, J. L., Thompson, S. E., et al. 2015, ApJS, 217, 31
Parviainen, H., \& Aigrain, S. 2015, MNRAS, 453, 3821

Paxton, B., Bildsten, L., Dotter, A., et al. 2011, ApJS, 192, 3

Paxton, B., Cantiello, M., Arras, P., et al. 2013, ApJS, 208, 4

Paxton, B., Marchant, P., Schwab, J., et al. 2015, ApJS, 220, 15

Pecaut, M. J., \& Mamajek, E. E. 2013, ApJS, 208, 9

Petigura, E. A. 2015, PhD thesis, Univ. California

Petigura, E. A., Howard, A. W., \& Marcy, G. W. 2013, PNAS, 110, 19273

Petigura, E. A., Howard, A. W., Marcy, G. W., et al. 2017, AJ, 154, 107

Petigura, E. A., Schlieder, J. E., Crossfield, I. J. M., et al. 2015, ApJ, 811, 102

Pope, B. J. S., Parviainen, H., \& Aigrain, S. 2016, MNRAS, 461, 3399

Rodriguez, D. R., Zuckerman, B., Kastner, J. H., et al. 2013, ApJ, 774, 101

Rogers, L. A. 2015, ApJ, 801, 41

Schlieder, J. E., Crossfield, I. J. M., Petigura, E. A., et al. 2016, ApJ, 818, 87

Storn, R., \& Price, K. 1997, Journal of Global Optimization, 11, 341

Van Cleve, J. E., Christiansen, J. L., Jenkins, J. M., et al. 2016, Kepler Science Document, KSCI-19040-005, ed. D. Caldwell et al., 2

Vanderburg, A., \& Johnson, J. A. 2014, PASP, 126, 948

Vanderburg, A., Johnson, J. A., Rappaport, S., et al. 2015, Natur, 526, 546

Vogt, S. S., Allen, S. L., Bigelow, B. C., et al. 1994, Proc. SPIE, 2198, 362

Weiss, L. M., \& Marcy, G. W. 2014, ApJL, 783, L6

Yee, S. W., Petigura, E. A., \& von Braun, K. 2017, ApJ, 836, 77 